Research Article

\title{
Neighborhoods and Manifolds of Immersed Curves
}

\author{
Andrea C. G. Mennucci 1 \\ Scuola Normale Superiore, Pisa, Italy \\ Correspondence should be addressed to Andrea C. G. Mennucci; andrea.mennucci@sns.it
}

Received 14 September 2021; Accepted 20 November 2021; Published 31 December 2021

Academic Editor: Luca Vitagliano

Copyright ( 2021 Andrea C. G. Mennucci. This is an open access article distributed under the Creative Commons Attribution License, which permits unrestricted use, distribution, and reproduction in any medium, provided the original work is properly cited.

We present some fine properties of immersions $\mathscr{I}: M \longrightarrow N$ between manifolds, with particular attention to the case of immersed curves $c: S^{1} \longrightarrow \mathbb{R}^{n}$. We present new results, as well as known results but with quantitative statements (that may be useful in numerical applications) regarding tubular coordinates, neighborhoods of immersed and freely immersed curve, and local unique representations of nearby such curves, possibly "up to reparameterization." We present examples and counterexamples to support the significance of these results. Eventually, we provide a complete and detailed proof of a result first stated in a 1991paper by Cervera, Mascaró, and Michor: the quotient of the freely immersed curves by the action of reparameterization is a smooth (infinite dimensional) manifold.

\section{Introduction}

In general, let $M$ and $N$ be smooth finite dimensional connected Hausdorff paracompact manifolds without boundary, with $\operatorname{dim}(M) \leq \operatorname{dim}(N)$.

This paper studies properties of immersions $\mathscr{I}: M \longrightarrow N$ that are $C^{1}$ maps such that $T \mathscr{I}_{x}$ is full rank at each $x$.

A particular but very interesting case are closed immersed curves $c: S^{1} \longrightarrow \mathbb{R}^{n}$ that are $C^{1}$ maps with $c^{\prime}(\theta) \neq 0$ at all $\theta \in S^{1}$, where $S^{1}=\left\{x \in \mathbb{R}^{2}:|x|=1\right\}$ be the circle in the plane. They will be called planar when $n=2$.

This paper is mostly devoted to this case (a forthcoming paper [15] will generalize many results in this paper to the general case of immersions $\mathscr{I}: M \longrightarrow N)$.

Immersed planar curves $c: S^{1} \longrightarrow \mathbb{R}^{2}$ have been used in computer vision for decades; indeed, the boundary of an object in an image can be modeled as a closed embedded curve, by the Jordan Theorem. Possibly, the first occurrence was active contours, introduced by [9] and used for the segmentation problem: the idea is to minimize energy, defined on contours or curves, that contains an image-based edge attraction term and a smoothness term, which becomes large when the curve is irregular. An evolution is derived to minimize the energy based on principles from the calculus of variations. There have been many variations to original model of [9]; for example [6] and a survey in [3].

An unjustified feature of the model of [9] was that the evolution is dependent on the way the contour is parameterized. Thereafter, the authors of $[4,11]$ considered minimizing a geometric energy, which is a generalization of Euclidean arclength, defined on curves for the edge-detection problem. The authors derived the gradient descent flow in order to minimize the geometric energy.

This lead to a principle: all operations related to curves should be independent of the choice of parameterizations.

Operations on the space of curves are best described and studied if the whole space of curves is endowed with a differential structure, so that it becomes a smooth manifold.

The above two remarks lead to the following question. If $I$ is the space of curves that we are interested in and $D$ is the action of reparameterization, then the quotient

$$
B=\frac{I}{D}
$$

is the space of curves up to parameterization (also called geometric curves in the following): when (and how) can we say that this quotient $B$ is a smooth manifold? 
This was discussed in [16], using a result from [5].

A purpose of this paper is to revisit the key result in [5]: indeed the proof in that paper is missing two key steps.

1.1. Plan of the Paper. In Section 2, we will define the needed topologies on the space of functions; we will present well known definitions and notations for curves, such as derivation and integration in arc parameter, length, normal vectors, and curvature; we will classify immersed and freely immersed curves and present results and examples.

In Section 3, we will present advanced results for immersed curves; we will discuss representation of nearby curves in tubular coordinates; we will show how the open neighborhood of a curve $c$ in the space of curves can be defined using tubular coordinates, so that if $c$ is immersed (respectively, freely immersed) then all curves in the neighborhood are immersed (respectively, freely immersed); we will show with examples what goes wrong when hypotheses are not met.

In Section 4, we will present the proof of this theorem: the quotient of the freely immersed curves by the action of reparameterization is a smooth (infinite dimensional) manifold. We will then explain, in a step by step analysis, why the original proof in [5] was incorrect.

A supplemental file contains Wolfram Mathematica code to generate some of the figures.

\section{Definitions}

In this section, we will present well-known definitions and results regarding immersions, with particular attention to immersed curves.

\subsection{Topologies}

Definition 1. We denote by $C^{r}(M, N)$ the space of all maps $f: M \longrightarrow N$ that are of class $C^{r}$. Here, $r \in\{0,1,2, \ldots, \infty\}$.

There are classically two types of topologies for this space.

(i) The weak topology, as defined in Ch. 1 Sec. 1 in [8], that coincides with the compact-open $C^{r}$-topology as defined in 41.9 in [12]; if $N=\mathbb{R}^{n}$, then the "weak topology" is the topology of the Fréchet space of local uniform convergence of functions and their derivatives up to order $r$

(ii) The strong topology as defined Ch. 1 Sec. 1 in [8] coincides with the Whitney $C^{r}$-topology as defined in 41.10 in [12]

If $M$ is compact, then the two above coincide; if moreover $N=\mathbb{R}^{n}$ and $r<\infty$, then $C^{r}\left(M, \mathbb{R}^{n}\right)$ is the usual Banach space.

Remark 1. If $N=\mathbb{R}^{n}$ but $M$ is not compact, then "strong topology" does not make $C^{r}\left(M, \mathbb{R}^{n}\right)$ a topological vector space since it has uncountably many connected components; but the connected component containing $f \equiv 0$ contains only compactly supported functions, and it has the topology $C_{c}^{r}\left(M, \mathbb{R}^{n}\right)$ (as defined in 6.9 in Rudin [18]) that is the strict inductive limit (for the definition of strict inductive limit and its properties, we refer to $17 \mathrm{G}$ at page 148 in [10]) of the immersions

$$
V_{K} \longrightarrow C_{c}^{r}\left(M, \mathbb{R}^{n}\right),
$$

where for each $K \subset M$ compact, $V_{K}$ is the space of $f: M \longrightarrow N$ that are zero outside of $K$, with a standard Banach (or Fréchet, for $r=\infty$ ) structure.

Proposition 1. The sets of immersions, submersions, and embeddings are open in $C^{r}(M, N)$ with the strong topology, for $r \geq 1$.

Proofs are in Ch. 1 Sec. 1 in [8].

Definition 2. For $r \in\{1, \ldots, \infty\}$, let $\operatorname{Diff}^{r}(M)$ be the family of diffeomorphisms of $M$ : all the maps $\phi: M \longrightarrow M$ that are $C^{r}$ and invertible, and the inverse $\phi^{-1}$ is $C^{r}$. It is a group, the group operation being "composition of functions."

Proposition 2. Diff ${ }^{r}(M)$ is open in $C^{r}(M, M)$ with the strong topology.

See Thm. 1.7 in Ch. 1 Sec. 1 in [8].

We will omit the superscript " $r$ " from $\operatorname{Diff}^{r}(M)$ in the following, for ease of notation.

\subsection{Immersions}

\subsubsection{Free Immersion}

Definition 3. An immersion $\mathscr{I}: M \longrightarrow N$ is called "free" if $\mathscr{I} \equiv \mathscr{I} \circ \phi$ for $\phi \in \operatorname{Diff}(M)$ implies that $\phi$ is the identity.

Proposition 3 (see [5] Lemma 1.3). If $\mathscr{I}$ is immersed and $\mathscr{I}(\phi(t))=\mathscr{I}(t)$ for all $t$ and $\phi(t)=t$ for a $t$, then $\phi=I d$.

Proof. Indeed, it is easily seen that

$$
\{t \in M: \phi(t)=t\}
$$

is closed; and it is also open, since an immersion is also a local diffeomorphism with its image.

As a corollary, if $\mathscr{I} \circ \phi \equiv \mathscr{I}$ and $\mathscr{I} \circ \psi \equiv \mathscr{I}$ and $\phi(t)=$ $\psi(t)$ for a $t$, then $\phi \equiv \psi$. Another corollary states the following.

Corollary 1 (see [5] Lemma 1.4). If $\mathscr{I}$ is an immersion and there is a $x \in \mathbb{R}^{n}$ s.t. $\mathscr{I}(t)=x$ for one and only one $t$, then $\mathscr{I}$ is a free immersion.

This implies that, when $\operatorname{dim}(M)<\operatorname{dim}(N)$, the free immersions are a dense subset of all immersions (for all the topologies considered in this paper). 
2.2.2. Reparameterizations and Isotropy Group. We first consider the general case of immersions $\mathscr{I}: M \longrightarrow N$.

Definition 4. The isotropy group (a.k.a. "stabilizer subgroup" or "little group") $\mathscr{G}_{\mathscr{I}}$ is the set of all $\phi \in \operatorname{Diff}(M)$ such that $\mathscr{I} \equiv \mathscr{I} \circ \phi$; it is a subgroup of $\operatorname{Diff}(M)$.

Obviously, $\mathscr{I}$ is freely immersed if and only if $\mathscr{G}_{\mathscr{I}}$ contains only the identity.

We will prove that $\mathscr{G}_{\mathscr{I}}$ is discrete and finite when $M$ is compact.

Remark 2. If we reparameterize $\tilde{\mathscr{I}}=\mathscr{I} \circ \widetilde{\varphi}$, then $\mathscr{G}_{1}$ changes by conjugation:

$$
\phi \in \mathscr{G}_{\mathscr{I}} \longleftrightarrow \widetilde{\varphi}^{-1} \circ \phi \circ \widetilde{\varphi} \in \mathscr{G}_{\tilde{I}} .
$$

Remark 3. If $M$ is orientable, then $\operatorname{Diff}(M)$ has a subgroup $\operatorname{Diff}^{+}(M)$ of orientation preserving diffeomorphisms; for the case of curves, then we obtain that $\operatorname{Diff}\left(S^{1}\right)$ has two connected components

$$
\operatorname{Diff}\left(S^{1}\right)=\operatorname{Diff}^{+}\left(S^{1}\right) \cup \operatorname{Diff}^{-}\left(S^{1}\right),
$$

where

(i) $\operatorname{Diff}^{+}\left(S^{1}\right)$ is the family of diffeomorphisms with $\phi^{\prime}>0$, and is a normal subgroup

(ii) $\operatorname{Diff}^{-}\left(S^{1}\right)$ is the family of diffeomorphisms with $\phi^{\prime}<0$

Consider a curve $c$ and let $\mathscr{G}_{c}$ be its isotropy group: we will prove in Lemma 3 that if $\phi \in \mathscr{G}_{c}$, then $\phi \in \operatorname{Diff}^{+}\left(S^{1}\right)$.

We will mostly use $\operatorname{Diff}^{+}\left(S^{1}\right)$ in the following.

Note that $\operatorname{Diff}^{+}\left(S^{1}\right)$ is a perfect group [19] (see [13] for a self-contained presentation); it is also a simple group: see Discussion in Sec. 2 in [2] for further references. (The author thanks Prof. Kathryn Mann for her help on these subjects.)

2.3. Curves. Remember that $S^{1}=\left\{x \in \mathbb{R}^{2}:|x|=1\right\}$ is the circle in the plane. We will often associate $\mathbb{R}^{2}=\mathbb{C}$, for convenience. In this case, we will associate $S^{1}=\left\{e^{i t}, t \in \mathbb{R}\right\} \subset \mathbb{C}$.

Definition 5. A closed curve is a map $c: S^{1} \longrightarrow \mathbb{R}^{n}$. We will always assume that the curve is of class $C^{1}$ (at least). The image of the curve, or trace of the curve, is $c\left(S^{1}\right)$.

When convenient, we will (equivalently) view $S^{1}$ as $\mathbb{R} /(2 \pi)$ (that is, $\mathbb{R}$ modulus $2 \pi$ translations), and consequently a closed curve will be a map $c: \mathbb{R} \longrightarrow \mathbb{R}^{n}$ that is $2 \pi$-periodic.

In particular, this will be the correct interpretation when we will write the operation $\theta_{1}+\theta_{2}$ for $\theta_{1}, \theta_{2} \in S^{1}$.

Remark 4. The "distance" of points in $S^{1}$ will be the intrinsic distance; this distance will be represented by the notation:

$$
d_{S^{1}}\left(\theta_{1}, \theta_{2}\right)
$$

for $\theta_{1}, \theta_{2} \in S^{1}$, and it is the length of the shortest arc in $S^{1}$ connecting the two points $\theta_{1}, \theta_{2}$. Note that if we identify $S^{1}$ to $\mathbb{R} /(2 \pi)$ and pick two points $\theta_{1}, \theta_{2} \in \mathbb{R} /(2 \pi)$ and represent them as real numbers, it may happen that

$$
\left|\theta_{1}-\theta_{2}\right|>d_{S^{1}}\left(\theta_{1}, \theta_{2}\right)
$$

Definition 6. (basepoint). We will select a distinguished point $\theta_{0}$ in the circle $S^{1}$ : for $S^{1} \subset \mathbb{R}^{2}$, it will be $\theta_{0}=(1,0)$; for $S^{1} \subset \mathbb{C}$, it will be $\theta_{0}=1$; for $S^{1}=\mathbb{R} /(2 \pi)$, it will be $\theta_{0}=0$.

Given a curve as above, we will call $c\left(\theta_{0}\right)$ the basepoint for the curve.

Example 1 (of a nonfreely immersed curve). The doubly traversed circle defined as

(i) $c_{2}(z)=z^{2}$ for $z \in S^{1}$ when we consider $S^{1} \subset \mathbb{C}$, or equivalently

(ii) $c_{2}(\theta)=(\cos (2 \theta), \sin (2 \theta))$ for $\theta \in \mathbb{R} /(2 \pi)$ that we identify with $S^{1}$

Setting $\phi(t)=t+\pi$, we have that $c_{2}=c_{2} \circ \phi$, so $c_{2}$ is not freely immersed.

Example 2 (taken from [5]). Note that there are free immersions without a point with only one preimage: consider a "figure eight" which consists of two touching circles. Now, we may map the circle to the figure eight by going first three times around the upper circle, then twice around the lower one. This immersion $c: S^{1} \longrightarrow \mathbb{R}^{2}$ is free.

We provide a simple Example 3 that shows how such curve can be made smooth.

2.3.1. Length, Tangent, and Curvatures. In the following, let $c: S^{1} \longrightarrow \mathbb{R}^{n}$ be an immersed curve.

Definition 7. If the curve $c$ is immersed, we can define the derivation with respect to the arc parameter

$$
\frac{\partial}{\partial s}=\frac{1}{\left|c^{\prime}\right|} \frac{\partial}{\partial \theta} \text {. }
$$

We will write $(\partial / \partial c)$ instead of $(\partial / \partial s)$ when we are dealing with multiple curves, and we will want to specify which curve is used.

Definition 8. We define the tangent vector

$$
T(\theta)=\frac{c^{\prime}(\theta)}{\left|c^{\prime}(\theta)\right|}=\frac{\partial}{\partial s} c(\theta) .
$$

Definition 9. The length of the curve $c$ is

$$
\operatorname{len}(c) \stackrel{\text { def }}{=} \int_{S^{1}}\left|c^{\prime}(\theta)\right| \mathrm{d} \theta .
$$


Definition 10. We define the integration by arc-parameter of a function $g: S^{1} \longrightarrow \mathbb{R}^{n}$ along the curve $c$ by

$$
\int_{c} g(s) \mathrm{d} s \stackrel{\text { def }}{=} \int_{S^{1}} g(\theta)\left|c^{\prime}(\theta)\right| \mathrm{d} \theta .
$$

There are two different definitions of curvature of an immersed curve: mean curvature $H$ and signed curvature $\kappa$, which is defined when $c$ is valued in $\mathbb{R}^{2}$.

$H$ and $k$ are extrinsic curvatures, they are properties of the embedding of $c$ into $\mathbb{R}^{n}$.

Definition $11(\mathrm{H})$. If $c$ is $C^{2}$ regular and immersed, we can define the (mean) curvature $H$ of $c$ as

$$
H=\frac{\partial}{\partial s} \frac{\partial}{\partial s} c=\frac{\partial}{\partial s} T
$$

It is easy to prove that $H \perp T$.

Definition $12(\mathrm{~N})$. When the curve $c$ is planar, we can define a normal vector $N$ to the curve, by requiring that $|N|=1$, $N \perp T$ and $N$ is rotated $\pi / 2$ degree anticlockwise with respect to $T$.

Definition 13. ( $\kappa$ ) If $c$ is in $\mathbb{R}^{2}$ and $C^{2}$, then we can define a signed scalar curvature $\kappa=\langle H, N\rangle$, so that

$$
\begin{aligned}
& \frac{\partial}{\partial s} T=\kappa N=H, \\
& \frac{\partial}{\partial s} N=-\kappa T .
\end{aligned}
$$

There is a choice of sign in the above two definitions; this choice agrees with the choice in [21].

When we will be dealing with multiple curves, we will specify the curve as a subscript, e.g., $T_{c}, \kappa_{c}, N_{c}$ will be the tangent, curvature, and normal to the curve $c$.

Remark 5. Note that $T, \kappa, N, H$ are geometrical quantities. If $\psi \in \operatorname{Diff}^{+}\left(S^{1}\right)$ and $\widetilde{c}=c \circ \psi$, then $T_{\widetilde{c}}=T_{c} \circ \psi, \kappa_{\tilde{c}}=\kappa_{c} \circ \psi$, $N_{\tilde{c}}=N_{c} \circ \psi$ and $H_{\tilde{c}}=H_{c} \circ \psi$.

2.3.2. Arc Parameter. Let $c$ be an immersed planar curve. We recall this important transformation.

Lemma 1 (constant speed reparameterization). A curve $c \in C^{1}$ can be reparameterized to $\tilde{c}=c \circ \varphi$ using a $\varphi \in \operatorname{Diff}^{+}\left(S^{1}\right)$ so that $\left|\widetilde{c}^{\prime}\right| \equiv \ell$ where $\ell=\operatorname{len}(c) /(2 \pi)$ is constant.

Proof. For simplicity, we assume that $S^{1}=[0,2 \pi]$. Let $L=$ len $(c)$, let $\quad \psi(t)=(2 \pi / L) \int_{0}^{t} \mid c^{\prime}(\theta) \| \mathrm{d} \theta$. Then $\psi:[0,2 \pi] \longrightarrow[0,2 \pi]$ is a diffeomorphism, let $\varphi=\psi^{-1}$.

Reparameterization to constant speed is a smooth operation in the space of curves, see Theorem 7 in [20].
When $\left|c^{\prime}\right| \equiv 1$, we will say that the curve is by arc $p a$ rameter. A curve can be reparameterized to arc parameter without changing its domain (as done above) iff len $(c)=2 \pi$ (if this is not the case, we will rescale the curve to make it so.)

\subsubsection{Angle Function and Rotation Index}

Proposition 4 (angle function and rotation index). If $c \in C^{1}$ is planar and is immersed, then $T=c^{\prime} /\left|c^{\prime}\right|$ is continuous and $|T|=1$, so there exists a continuous function $\alpha: \mathbb{R} \longrightarrow \mathbb{R}$ satisfying

$$
T(s)=(\cos (\alpha(s)), \sin (\alpha(s))),
$$

and $\alpha(s)$ is unique, up to adding the constant $k 2 \pi$ with $k \in \mathbb{Z}$. $\alpha$ is called the angle function.

Moreover $\alpha(s+2 \pi)-\alpha(s)=2 \pi I$, where $I$ is an integer, known as rotation index of $c$. This number is unaltered if $c$ is deformed by a smooth homotopy (Figure 1).

See 2.1.4 in [1] or Theorem 53.1 in [17], and following.

Remark 6. We can use the angle function to compute the scalar curvature $\kappa$, that was defined in Definition 13 by $\partial_{s} T=\kappa N$, indeed deriving (14) and combining this with

$$
N(s)=(-\sin (\alpha(s)), \cos (\alpha(s))),
$$

we obtain

$$
\kappa=\frac{\alpha^{\prime}}{\left|c^{\prime}\right|}=\partial_{s} \alpha
$$

2.4. Shapes. Shapes are usually considered to be geometric objects. Representing a curve using $c: S^{1} \longrightarrow \mathbb{R}^{n}$ forces a choice of parameterization that is not really part of the concept of "shape."

Suppose that $I$ is a space of immersed curves c: $S^{1} \longrightarrow \mathbb{R}^{n}$.

Definition 14 (geometric curves). The quotient space $B=$ $I / \operatorname{Diff}\left(S^{1}\right)$ is the space of curves up to reparameterization, also called geometric curves in the following. Two parametric curves $c_{1}, c_{2} \in M$ such that $c_{1}=c_{2} \circ \phi$ for a $\phi \in \operatorname{Diff}\left(S^{1}\right)$ are the same geometric curve inside $B$.

$B$ is mathematically defined as the set $B=\{[c]\}$ of all equivalence classes $[c]$ of curves that are equal but for reparameterization,

$$
[c] \stackrel{\text { def }}{=}\left\{c \circ \phi, \text { for } \phi \in \operatorname{Diff}\left(S^{1}\right)\right\} .
$$

We may also consider the quotient w.r.t $\operatorname{Diff}^{+}\left(S^{1}\right)$. The quotient space I/Diff ${ }^{+}\left(S^{1}\right)$ is the space of geometric-oriented curves.

Unfortunately, the quotient of immersed curves by reparameterizations is not a manifold; but the quotient of freely immersed curve is. 


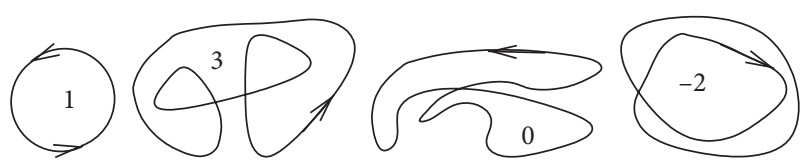

FIgURE 1: Examples of curves of different rotation index.

Theorem 1. Suppose that I is the space of the freely immersed curves; and that I and Diff $\left(S^{1}\right)$ have the topology of the Fréchet space of $C^{\infty}$ functions, then the quotient $B=I /$ Diff $^{+}\left(S^{1}\right)$ is a smooth manifold modeled on $C^{\infty}$.

One aim of this paper will be to give a complete proof of this result, first presented in [5]. (We remark that the theorem in [5] was presented for the case of immersions $\mathscr{I}: M \longrightarrow N)$. The proof is in Section 4.1. Indeed, as we will discuss in Section 4.2, the proof in [5] misses some key arguments.

\section{Advanced Properties of Immersed Curves}

In this section, we will present results regarding immersed curves that are either new or presented in more precise form than usually found in the literature.

Most of the results are presented, for sake of simplicity, for planar curves $c: S^{1} \longrightarrow \mathbb{R}^{2}$, but can be extended to the case of curves $c: S^{1} \longrightarrow N$ taking values in a manifold $N$, up to some nuisance in notations.

The general case of immersions $\mathscr{I}: M \longrightarrow N$ requires instead some arguments that will be discussed in a future paper [15].

\subsection{Examples}

Definition 15. We start with some classical examples of $C^{\infty}$ functions of compact support. Let

$$
\begin{gathered}
\eta_{0}: \mathbb{R} \longrightarrow \mathbb{R}, \quad \eta_{0}(u) \stackrel{\text { def }}{=} \begin{cases}e^{(4 / \pi)-(1 / u)-(1 /(\pi-u))}, & u \in[0, \pi], \\
0, & \text { elsewhere, }\end{cases} \\
\eta_{1}: \mathbb{R} \longrightarrow \mathbb{R}, \quad \eta_{1}(u) \stackrel{\text { def }}{=} \begin{cases}4(2 u-\pi) e^{-(1 / u)-(1 /(\pi-u))}, & u \in[0, \pi], \\
0, & \text { elsewhere, }\end{cases}
\end{gathered}
$$

(see Figure 2 on the following page).

We will use these to build some following examples.

Example 3. We present here a simple smooth formula for Example 2

$$
\begin{aligned}
& c:[0,5 \pi] \longrightarrow \mathbb{R}^{2}, \\
& c(u) \stackrel{\text { def }}{=}\left(\sin (2 u), c_{2}(u)\right), \\
& c_{2}(u) \begin{cases}\eta_{0}(u), & u \leq \pi, \\
\eta_{0}(u-\pi), & u \leq 2 \pi, \\
-\eta_{0}(u-2 \pi), & u \leq 3 \pi, \\
-\eta_{0}(u-3 \pi), & u \leq 4 \pi, \\
-\eta_{0}(u-4 \pi), & u \leq 5 \pi .\end{cases}
\end{aligned}
$$

this is a $C^{\infty}$ function depicted at Figure 3 on the next page.

3.1.1. Trace and Parameterization. If a curve is embedded then the curve is identified by its image, in these senses.

(i) If $c, \widetilde{c}: S^{1} \longrightarrow \mathbb{R}^{n}$ are embedded and have the same image, then there is an unique reparameterization $\phi$ such that $\widetilde{c}=c \circ \phi$.

(ii) Suppose that $c_{0}: S^{1} \longrightarrow \mathbb{R}^{n}$ is embedded and $A=$ $c\left(S^{1}\right)$ is the trace; suppose that $c_{0}$ is parameterized by constant speed parameter; let us fix a candidate basepoint $v \in A$ in the trace.
We can state that $A, v$ characterize the embedded curve up to a choice of direction: precisely, there are exactly two different $c_{1}, c_{2}: S^{1} \longrightarrow \mathbb{R}^{n}$, parameterized by constant speed parameter, such that $c_{1}\left(\theta_{0}\right)=c_{2}\left(\theta_{0}\right)=v$, and they satisfy

$$
\begin{aligned}
& c_{1}(\theta)=c_{0}(\theta+a), \\
& c_{2}(\theta)=c_{0}(b-\theta),
\end{aligned}
$$

for unique choices of $a, b \in S^{1}$ (dependant on $v$ ).

In particular, if the rotation index of $c_{0}$ is $r$, then the latter curves have rotation indexes $\pm r$.

Since the definition of freely immersed curve says that the curve identifies a unique parameterization, then we may be induced to think that the above two properties extend to freely immersed curves: but this is not the case.

Example 4. The following two curves have the same trace, are freely immersed, are smooth, but have rotation indexes 0 and 1.

(1) This immersed closed curve $c:[0,2 \pi] \longrightarrow \mathbb{R}^{2}$ with components

$$
\begin{aligned}
& c_{1}(u)=\sin (2 u), \\
& c_{2}(u)= \begin{cases}\eta_{1}(2 u), & u \in\left[0, \frac{\pi}{2}\right], \\
\eta_{1}\left(2 u-\frac{\pi}{2}\right), & u \in\left[\frac{\pi}{2}, \pi\right] .\end{cases}
\end{aligned}
$$



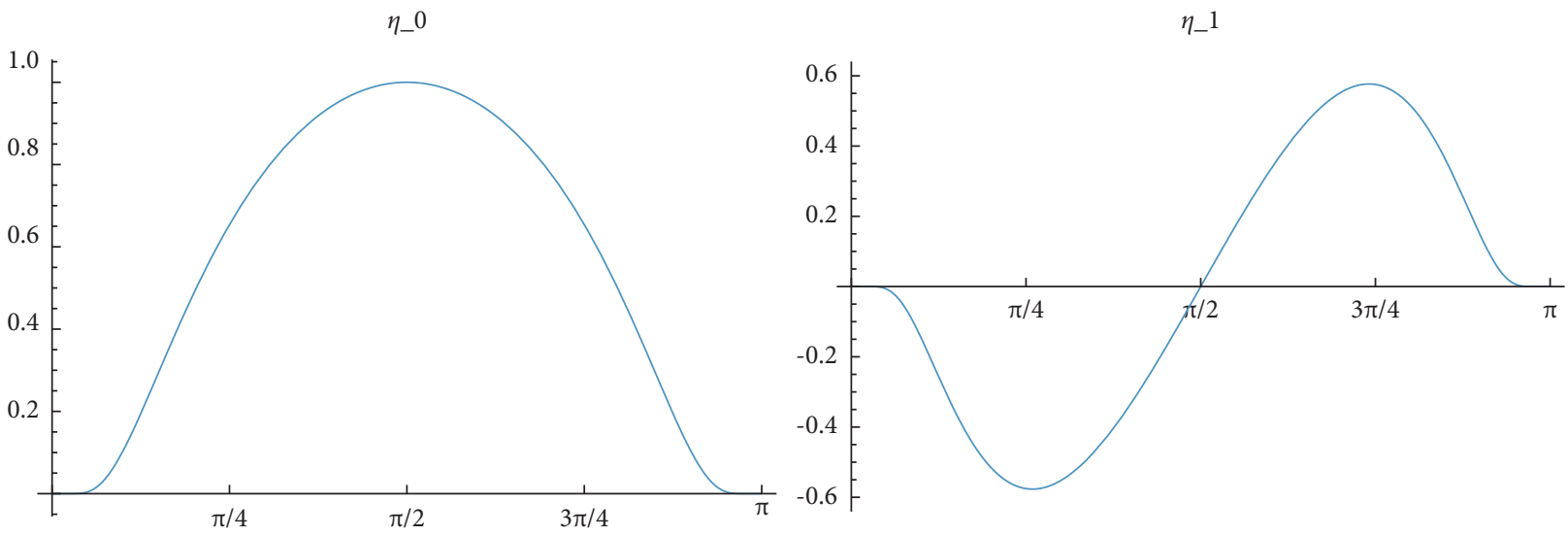

Figure 2: Graph of $\eta_{0}$ as defined in (18); graph of $\eta_{1}$ as defined in (19).

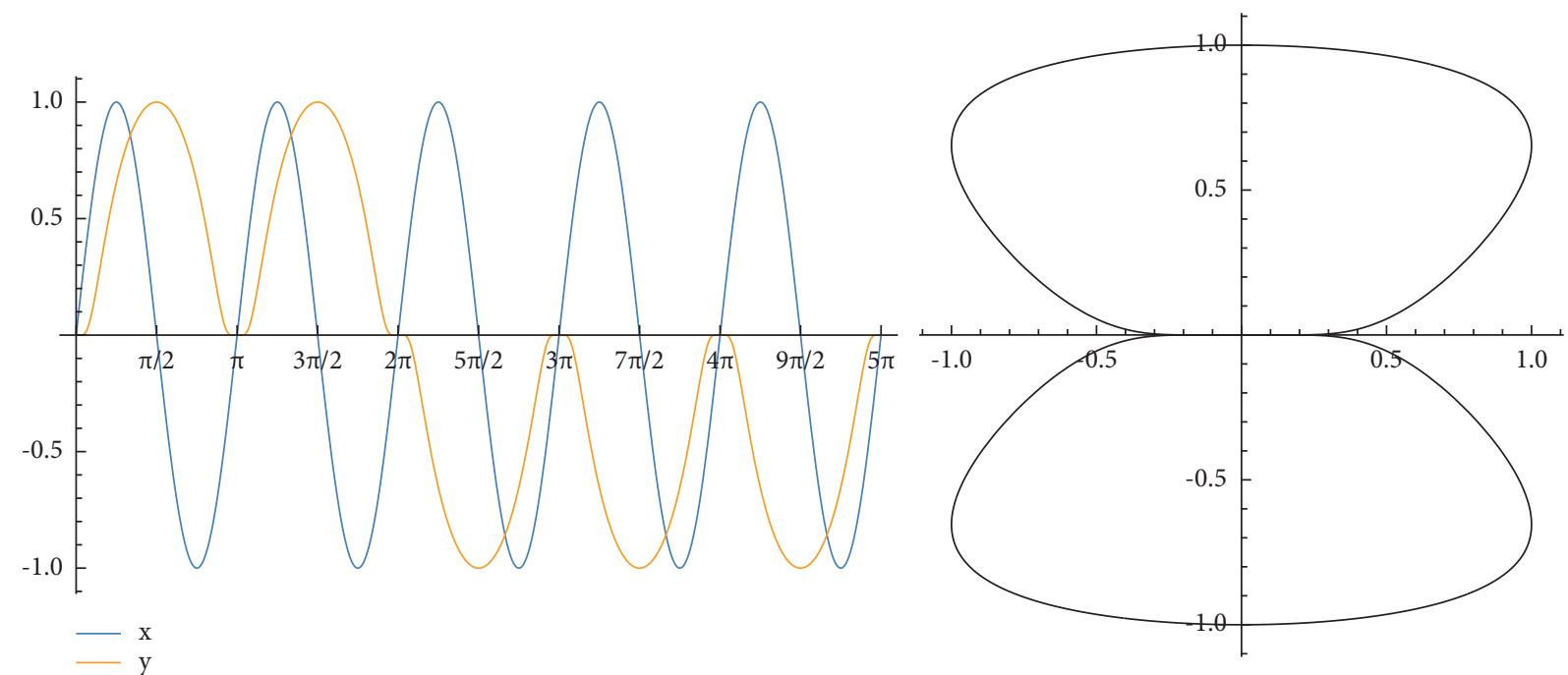

Figure 3: $x$ and $y$ coordinates of curve defined in Example 2; trace of curve defined in Example 2.

(2) This immersed closed curve $c:[0,2 \pi] \longrightarrow \mathbb{R}^{2}$ with components

$$
\begin{aligned}
& c_{2}(u)=\sin (2 u), \\
& c_{2}(u)= \begin{cases}\eta_{1}(2 u), & u \in\left[0, \frac{\pi}{2}\right], \\
-\eta_{1}\left(2 u-\frac{\pi}{2}\right), & u \in\left[\frac{\pi}{2}, \pi\right] .\end{cases}
\end{aligned}
$$

See Figure 4 on the following page.

\subsection{Reparameterizations and Isotropy Group}

Lemma 2. If $\phi \in \operatorname{Diff}^{-}\left(S^{1}\right)$, then $\phi$ has two fixed points.

Proof. We represent $\phi$ as a map $\phi:[0,2 \pi] \longrightarrow \mathbb{R}$ that is continuous, strictly decreasing and such that

$$
\begin{gathered}
\phi(0) \in[2 \pi, 4 \pi), \\
\phi(2 \pi)=\phi(0)-2 \pi,
\end{gathered}
$$

then

$$
\phi(2 \pi) \in[0,2 \pi)
$$

so the graph $y=\phi(x)$ must intersect both the graph $y=x$ and the graph $y=x+2 \pi$ for two different points $x_{1}, x_{2} \in[0,2 \pi)$ that are the two fixed points.

Lemma 3. If $c$ is immersed and $\psi \in \mathscr{G}_{c}$, then $\psi \in \operatorname{Diff}^{+}\left(S^{1}\right)$.

Proof. Suppose that $\psi \in \operatorname{Diff}^{-}\left(S^{1}\right)$, let $u \in S^{1}$ be a fixed point (by Lemma 2). By deriving

$$
c^{\prime}(\theta)=c^{\prime}(\psi(\theta)) \psi^{\prime}(\theta),
$$

setting $\theta=u$

$$
c^{\prime}(u)=c^{\prime}(u) \psi^{\prime}(u)
$$

and this is impossible since $\psi^{\prime}(u)<0$. 


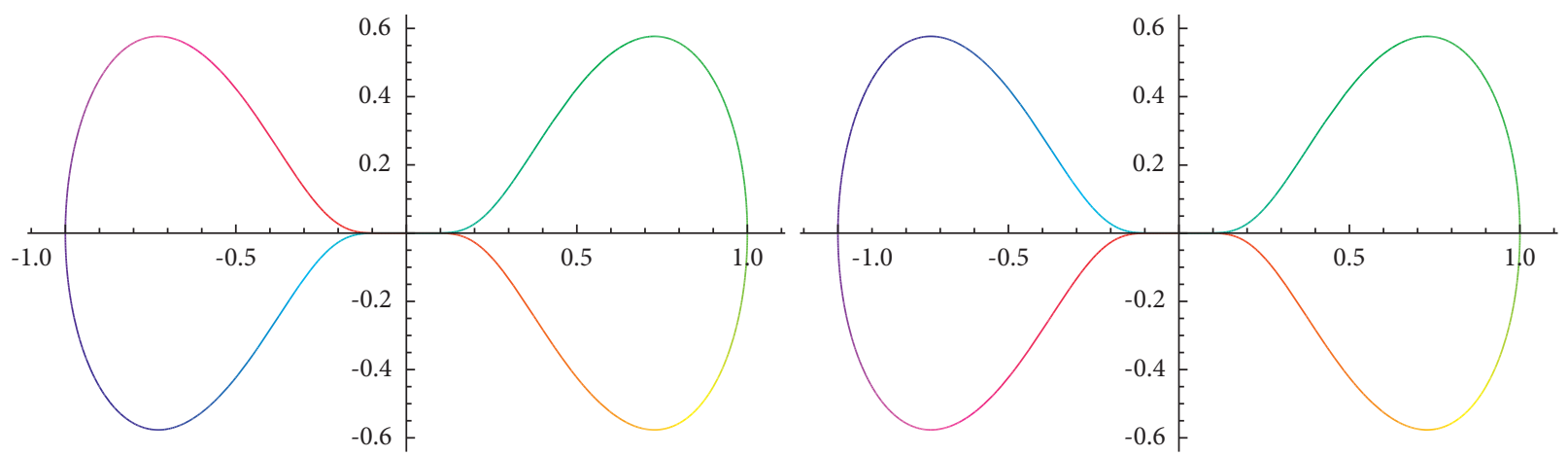

Two smooth curves with the same trace.
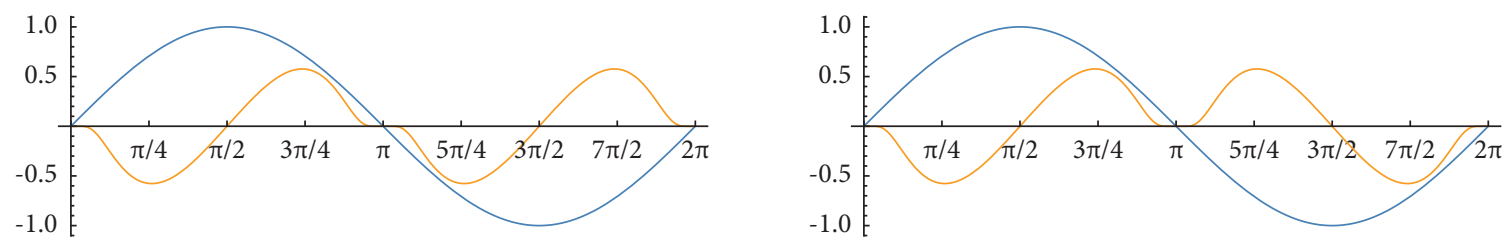

Their respective parameterizations.
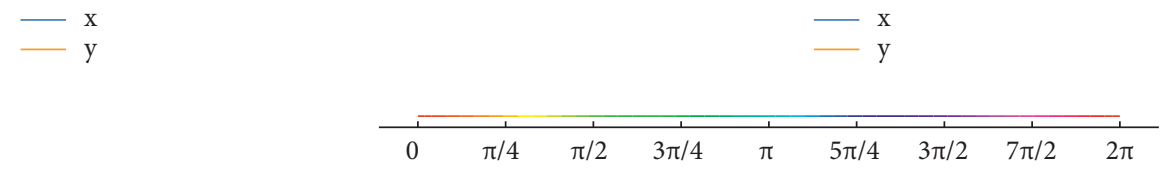

FIgURE 4: Trace of the two curves and parameterization defined in Example 4; with color scheme used for the parameter.

\subsection{Local Embedding}

\subsubsection{Length of Curve Arcs}

Definition 16. Suppose $c: S^{1} \longrightarrow \mathbb{R}^{n}$ is $C^{1}$. Let $\sigma, \widetilde{\sigma} \in S^{1}$. When $\sigma \neq \tilde{\sigma}$ there are two arcs in $S^{1}$ connecting $\sigma$ to $\widetilde{\sigma}$. By

$$
\text { len } c_{\mid[\sigma, \widetilde{\sigma}]} \text {, }
$$

we will mean the minimum of the lengths of $c$ when restricted to one of the two arcs connecting $\sigma$ to $\widetilde{\sigma}$.

If $c$ is periodically extended to $c: \mathbb{R} \longrightarrow \mathbb{R}^{n}$ and $\sigma, \widetilde{\sigma} \in \mathbb{R}$, then there is an unique $k \in \mathbb{Z}$ such that

$$
\sigma \leq \tilde{\sigma}+k 2 \pi<\sigma+2 \pi
$$

and then, letting

$$
\begin{aligned}
& l_{1}=\int_{\sigma}^{\tilde{\sigma}+k 2 \pi}\left|c^{\prime}(\theta)\right| \mathrm{d} \theta, \\
& l_{2}=\int_{\tilde{\sigma}+k 2 \pi}^{\sigma+2 \pi}\left|c^{\prime}(\theta)\right| \mathrm{d} \theta,
\end{aligned}
$$

we define

$$
\text { len } c_{\mid[\sigma, \widetilde{\sigma}]}=\min \left\{l_{1}, l_{2}\right\} \text {. }
$$

In particular, when $c$ is parameterized at constant speed (i.e., $\left|c^{\prime}\right| \equiv \ell$ ), then we will (covertly) assume that $\sigma$ and $\widetilde{\sigma}$ are chosen (up to adding $k 2 \pi$ ) so that $|\sigma-\widetilde{\sigma}|=d_{S^{1}}(\sigma, \widetilde{\sigma}) \leq \pi$ and then

$$
\operatorname{len} c_{\mid[\sigma, \widetilde{\sigma}]}=\ell|\sigma-\tilde{\sigma}|
$$

Remark 7. When $c$ is not parameterized by constant velocity, the above may lead to some confusion. The interval $[\sigma, \tilde{\sigma}]$ in the notation (28) implicitly refers to the choice of $\operatorname{arc}$ in $S^{1}$ that provides the above minimum. Note that this may not be the shortest arc connecting $\sigma$ to $\widetilde{\sigma}$ in $S^{1}$. This may happen if the parameterization of $c$ has regions of fast and slow velocity, as in this example.

Example 5. Let $\widetilde{c}(\theta)=(\cos (\theta), \sin (\theta))$ be the standard circle, and

$$
\phi(\theta)= \begin{cases}4 \theta, & 0 \leq \theta \leq \frac{\pi}{3}, \\ \frac{2}{5}(x+3 \pi), & \frac{\pi}{3} \leq \theta \leq 2 \pi,\end{cases}
$$

(see plot in Figure 5) then smooth out the corners of $\phi$ so that it becomes a diffeomorphism of $S^{1}$; let

$$
c=\tilde{c} \circ \phi,
$$

let $\theta_{0}=0, \theta_{1}=\pi / 3$ in $S^{1} \sim \mathbb{R} /(2 \pi)$, then $d_{S^{1}}\left(\theta_{0}, \theta_{1}\right)=\pi / 3$, and is given by the arc moving counterclockwise from $\theta_{0}$ to $\theta_{1}$, while

$$
\text { len } c_{\mid\left[\sigma_{0}, \sigma_{1}\right]}=\frac{2 \pi}{3}
$$




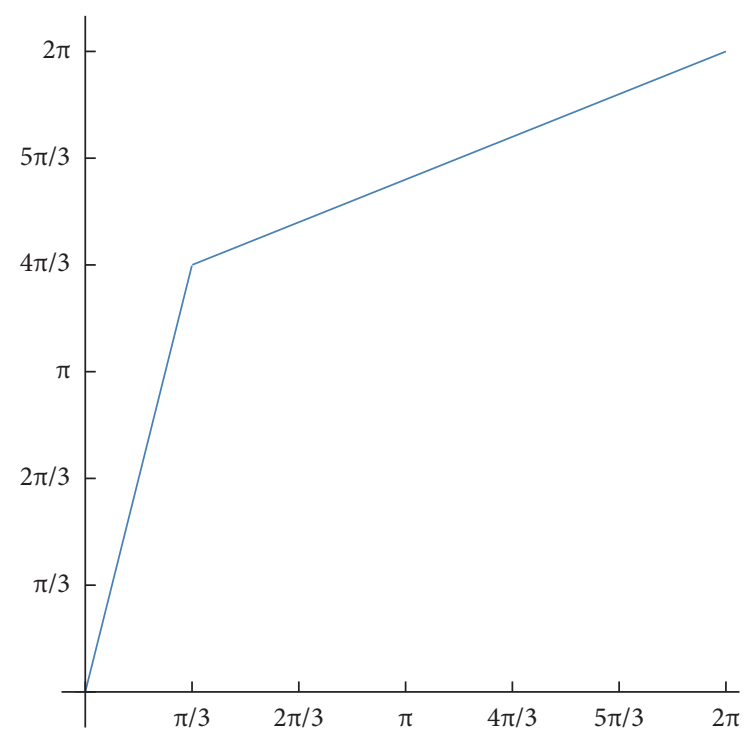

FIgURE 5: Plot of function $\phi$ in equation (33) in Example 5.

is given by the arc moving clockwise from $\theta_{0}$ to $\theta_{1}$.

This never happens for small distances/lengths, though.

Theorem 2. Fix an immersed curve $c: S^{1} \longrightarrow \mathbb{R}^{n}$; let

$$
\begin{aligned}
& M=\max \left|c^{\prime}\right|, \\
& m=\min \left|c^{\prime}\right|, \\
& \sigma_{c} \stackrel{\text { def }}{=} \frac{1}{(M / m)+1} .
\end{aligned}
$$

(i) For any $\theta_{0}, \theta_{1}$ in $S^{1}$ such that

$$
d_{S^{1}}\left(\theta_{0}, \theta_{1}\right)<2 \pi \sigma_{c}
$$

the shortest arc connecting them in $S^{1}$ is also the arc where

$$
\operatorname{len} c_{\mid}\left[\theta_{0}, \theta_{1}\right]
$$

is computed

(ii) For any $\theta_{0}, \theta_{1}$ in $S^{1}$ such that

$$
\text { len } c_{\mid\left[\theta_{0}, \theta_{1}\right]}<\sigma_{c} \operatorname{len}(c)
$$

the arc where

$$
\text { len } c_{\mid\left[\theta_{0}, \theta_{1}\right]} \text {, }
$$

is computed is also the shortest arc connecting them in $S^{1}$, whose length is

$$
d_{S^{1}}\left(\theta_{0}, \theta_{1}\right) \text {. }
$$

(iii) In any of the above cases,

$$
m d_{S^{1}}\left(\theta_{0}, \theta_{1}\right) \leq \operatorname{len} c_{\mid\left[\theta_{0}, \theta_{1}\right]} \leq M d_{S^{1}}\left(\theta_{0}, \theta_{1}\right)
$$

\subsubsection{Estimates. We begin with this estimate.}

Proposition 5. Let $\alpha(s)$ be the angle function, for $s \in J=[0,2 \pi]$. The fact that the curve is closed imposes lower bounds on $\max _{J}\left|\alpha^{\prime}\right|$.

(i) If the rotation index $\mathscr{I}$ of the curve is not zero, then $|\alpha(2 \pi)-\alpha(0)|=2 \pi|I|$ so necessarily $\max _{J}\left|\alpha^{\prime}\right| \geq|I|$

(ii) If the rotation index of the curve is zero, then necessarily $\max _{J}\left|\alpha^{\prime}\right| \geq 1 / 2$

Indeed, we can prove that

$$
\max _{J} \alpha-\min _{J} \alpha \geq \pi
$$

otherwise, let

$$
\beta=\frac{\max _{J} \alpha+\min _{J} \alpha}{2},
$$

taking $v=(\cos \beta, \sin \beta)$, we would have

$$
v \cdot c^{\prime}(\theta)=\left|c^{\prime}(\theta)\right| \cos (\beta-\alpha(\theta))>0,
$$

for all $\theta$, hence the curve would not be closed.

Definition 17. Given $c$ : $S^{1} \longrightarrow \mathbb{R}^{2}$, a $C^{2}$ immersed closed curve, we recall that $\kappa$ is the scalar curvature of $c$; we define

$$
\begin{gathered}
\delta_{c} \stackrel{\text { def }}{=} \frac{\pi}{(3 \max |\kappa|)}, \\
\tau_{c} \stackrel{\text { def }}{=} \frac{1}{(2 \max |\kappa|)} .
\end{gathered}
$$

Note that since the curve is closed, $\kappa$ cannot be identically zero.

Note that $\delta_{c}=\tau_{c}(2 \pi / 3)$, but we define two quantities since this simplifies the notation in the following. We have $2 \tau_{c} \leq \delta_{c}$ but $3 \tau_{c} \geq \delta_{c}$.

Remark 8. Note that if we rescale the curve $c$ by a factor $\lambda$, then $\delta_{c}, \tau_{c}$ and len $c_{\mid[\sigma, \tilde{\sigma}]}$ are multiplied by $\lambda$ as well. If we rotate or translate $c$, then $\delta_{c}$, $\tau_{c}$ and len $c_{\mid[\sigma, \tilde{\sigma}]}$ are unaffected. If we reparameterize, then $\delta_{c}, \tau_{c}$ are unchanged, whereas if $\psi \in \operatorname{Diff}\left(S^{1}\right)$ and $\widetilde{c}=c \circ \psi$, we have

$$
\operatorname{len} \widetilde{c}_{\mid[\psi(\sigma), \psi(\widetilde{\sigma})]}=\operatorname{len} c_{\mid[\sigma, \widetilde{\sigma}]} .
$$

In all (with the exception of relation (100) in Lemma 7) following definitions, propositions, and theorems, the formulas are built to be "geometrical": this means that, if the curves are reparameterized, rescaled, translated or rotated, then the formulas change in predictable ways (as explained above).

This simplifies the proofs: in the proofs we can assume, with no loss of generality, that the curve is parameterized by arc parameter.

Remark 9. Note that $\delta_{c} \leq$ len $(c) / 3$ for curves of index zero and $\delta_{c} \leq \operatorname{len}(c) /(6|I|)$ for curves of index $I \neq 0$. 
Proof. We use Proposition 5. The formula in the thesis is invariant for reparameterizations and scaling; we rescale the curve so that len $(c)=2 \pi$ and reparameterize by arc parameter so that $\left|c^{\prime}\right| \equiv 1$. For curves of index zero, the thesis $\pi /\left(3 \max _{J}|\kappa|\right) \leq \operatorname{len}(c) / 3$, that is, $\pi \leq \max _{J}|\kappa|$ len $(c)$; since $\left|c^{\prime}\right| \equiv 1$ by (16), this last becomes $1 / 2 \leq \max _{J}\left|\alpha^{\prime}\right|$ that was proved above. For curves of index $I$, the thesis $\pi /\left(3 \max _{J}|\kappa|\right) \leq \operatorname{len}(c) /(6|I|)$, that is, $2 \pi|I| \leq \max _{J}|\kappa|$ len $(c)$, then becomes $|I| \leq \max _{J}\left|\alpha^{\prime}\right|$ that was proved above.

For $I \neq 0$ the above is sharp, as in the case of $c(\theta)=(\cos (I \theta), \sin (I \theta))$.

3.3.3. Local Embedding of Curves. It is well known that a $C^{1}$ immersion $\mathscr{I}: M \longrightarrow N$ is a local embedding. For curves of class $C^{2}$, we can provide a simple quantitative statement.

Proposition 6 (local embedding). Let $c: S^{1} \longrightarrow \mathbb{R}^{2}$ a $C^{2}$ immersed curve. Define $\delta_{c}$ as in Definition 17. For any $a, b \in S^{1}$, let $L \stackrel{\text { def }}{=}$ len $c_{\mid[a, b]}$; assume that $L \leq 2 \delta_{c}$, then $|c(b)-c(a)| \geq L / 2$; so, $c_{\mid[a, b]}$ is embedded.

Proof. For simplicity, we assume that $c$ is periodically extended to $c: \mathbb{R} \longrightarrow \mathbb{R}^{2}$; then, we identify the interval in $\mathbb{R}$ that is associated to the arc of the curve where the length len $c_{\mid[a, b]}$ is computed; for simplicity, we call this interval $[a, b]$ again. (if the arc is short enough, then by Theorem 2, no ambiguity is possible).

Using Lemma 1 and Remark 8, assume that $\left|c^{\prime}\right| \equiv \ell=\operatorname{len}(c) /(2 \pi)$; then, $\partial_{s}=(1 / \ell) \partial_{\theta}$, so $T=c^{\prime} / \ell$ and

$$
\text { len } c_{\mid[a, b]}=\ell(b-a) \text {. }
$$

As noted in (16)

$$
|\kappa|=\left|\partial_{s} T\right|=\frac{\left|T^{\prime}\right|}{\ell}=\frac{\left|\alpha^{\prime}\right|}{\ell},
$$

so $\delta_{c}=\ell \pi /\left(3 \max \left|\alpha^{\prime}\right|\right)$. Let $m=(a+b) / 2$ be the middle point. Let $\alpha(t)$ be the angle function (14). Up to rotation, suppose $c^{\prime}(m)=(\ell, 0), T(m)=(1,0)$; so, we can assume $\alpha(m)=0$. Let $L=\operatorname{len} c_{\mid[a, b]}=\ell(b-a) \leq 2 \delta_{c}$. For any $\theta \in[a, b], \quad$ we have $\quad \ell|\theta-m| \leq \delta_{c}$; hence, $|\theta-m| \leq \pi /\left(3 \max \left|\alpha^{\prime}\right|\right)$; hence, for all $a \leq \theta \leq b$, we have

$$
|\alpha(\theta)| \leq|\theta-m| \max \left|\alpha^{\prime}\right| \leq \frac{\pi}{3}
$$

and hence, $\cos (\alpha(\theta)) \geq 1 / 2$; hence, for $a \leq \theta_{1} \leq \theta_{2} \leq b$, for the abscissa, we can write

$$
c_{1}\left(\theta_{2}\right)-c_{1}\left(\theta_{1}\right)=\ell \int_{\theta_{1}}^{\theta_{2}} \cos (\alpha(\theta)) \mathrm{d} \theta \geq \ell\left(\theta_{2}-\theta_{1}\right) \frac{1}{2} \text {. }
$$

3.4. Isotropy Group Is Discrete. Given an immersion $\mathscr{I}: M \longrightarrow N$, it is possible to prove that the isotropy group is discrete (when $M$ is paracompact) and even finite (when $M$ is compact; this latter result appears in [5]). When considering curves, we can obtain the same results (and even more) in a more direct and geometric way.
Lemma 4. Let $c: S^{1} \longrightarrow \mathbb{R}^{n}$ be immersed.

(i) $\mathscr{G}_{c}$ is finite

(ii) If $c \circ \psi \equiv c \circ \phi$ and $\psi(a)=\phi(a)$ for an $a \in S^{1}$, then $\psi \equiv \phi$

(iii) If $c$ is parameterized by constant speed (see Lemma $1)$, then there is a $k \in \mathbb{N}, k \geq 1$ s.t. $\mathscr{G}_{c}$ is the set of all $\phi(t)=t+(2 \pi j / k)$ for $j=0, \ldots, k-1$ (the proof of this is a special case of the 2nd step of the proof of Lemma 9)

Proof.

(i) We prove the third point. Indeed deriving $c=c \circ \phi$ and noting that $\left|c^{\prime}\right| \equiv \ell$, we obtain $\phi^{\prime} \equiv 1$ so $\phi(t)=t+\beta$; hence, $\phi^{j}(t)=t+j \beta \in \mathscr{G}_{c}$, for all $j$; if $\beta / \pi$ is irrational, then $j \beta$ would be dense in $S^{1}=\mathbb{R} /(2 \pi)$, and this is denied by Proposition 6 . Moreover, if $\phi(t)=t+(2 \pi / k)$ and $c=c \circ \phi$, then $c(0)=c(2 \pi / k)$; but by Proposition 6, $(2 \pi) /(3 \max |\kappa|) \leq(2 \pi \ell / k)$, that is, $k \leq 3 \ell \max |\kappa|$. So, there is an unique $k$ such that any $\phi \in \mathscr{G}_{c}$ can be written as $\phi(t)=t+(2 \pi j / k)$.

(ii) The above characterization shows that if $\phi^{\prime} \equiv 1 \equiv \psi^{\prime}$ and $\phi(a)=\psi(a)$, then $\phi \equiv \psi$. By Remark 2, this is valid for any curve (even when it is not parameterized by constant speed). This proves the second point.

(iii) The first point follows again from Remark 2.

3.5. Tubular Neighborhoods. Existence of tubular neighborhood is well known; we provide a quantitative result for $C^{2}$ planar immersed curves.

Proposition 7 (tubular neighborhood). Define $\delta_{c}, \tau_{c}$ as in Definition 17. Fix $a, b \in S^{1}$ with len $c_{\mid[a, b]} \leq 2 \delta_{c}$. Let

$$
\begin{gathered}
\Phi:[a, b] \times\left[-\tau_{c}, \tau_{c}\right] \longrightarrow \mathbb{R}^{2}, \\
\Phi(s, t)=c(s)+t N(s),
\end{gathered}
$$

then $\Phi$ is a diffeomorphism with its image. Moreover, if the $\operatorname{arc}\left[s_{1}, s_{2}\right]$ is contained in the arc $[a, b]$ identified above, then

$$
\left|\Phi\left(s_{1}, t_{1}\right)-\Phi\left(s_{2}, t_{2}\right)\right| \geq \frac{1}{4} \operatorname{len} c_{\mid\left[s_{1}, s_{2}\right]},
$$

whereas (obviously)

$$
\left|\Phi\left(s, t_{1}\right)-\Phi\left(s, t_{2}\right)\right|=\left|t_{2}-t_{1}\right| \text {. }
$$

Proof. Assume that the curve has length $2 \pi$ and is parameterized in arc parameter; with no loss of generality (recalling Remark 8); let $\alpha$ be the angle function (14).

Extend $c$ to a periodic function $c: \mathbb{R} \longrightarrow \mathbb{R}^{2}$ and identify the interval in $\mathbb{R}$ that is associated with the arc of the curve where the length len $c_{\mid[a, b]}$ is computed. For simplicity, we call this interval $[a, b]$ again (if the arc is short enough, then by Theorem 2, no ambiguity is possible). 
The Jacobian of $\Phi$ is

$$
\begin{aligned}
& \frac{\partial}{\partial s} \Phi=T(1-\kappa t), \\
& \frac{\partial}{\partial t} \Phi=N,
\end{aligned}
$$

so its determinant is $(1-\kappa t) \geq 1 / 2$ by the hypothesis $|t| \leq \tau_{c}$.

We will then prove that $\Phi$ is injective, so it will be an homeomorphism with its image, and since it is a local diffeomorphism, it will be a diffeomorphism.

Choose $\left(s_{1}, t_{1}\right)$ and $\left(s_{2}, t_{2}\right)$ with $a \leq s_{1}<s_{2} \leq b$ and $\left|t_{1}\right| \leq \tau_{c},\left|t_{2}\right| \leq \tau_{c}$.

We set $m=\left(s_{2}+s_{1}\right) / 2$. Up to rotation, we assume that $T(m)=e_{1}=(1,0)$ and $\alpha(m)=0$, so that $N(m)$ is perpendicular to the $x$ axis. As in Proposition 6, we can prove that $\cos (\alpha(s)) \geq 1 / 2$ for all $s_{1} \leq s \leq s_{2}$.

We write

$$
\Phi(s, t)=c(s)+t N(s)=c(m)+\int_{m}^{s} T(s) \mathrm{d} \theta+t N(s),
$$

and then for the abscissa

$$
\Phi(s, t)_{1}=c(m)_{1}+\int_{m}^{s} \cos (\alpha(\theta)) \mathrm{d} \theta+t \sin (\alpha(s)),
$$

note that $\Phi(m, t)_{1}=c(m)_{1}$. Deriving, we obtain

$$
\frac{\partial}{\partial s} \Phi(s, t)_{1}=\cos (\alpha(s))\left(1-t \alpha^{\prime}(s)\right) \geq \frac{1}{4} .
$$

We then obtain that

$$
\Phi\left(s_{2}, t_{2}\right)_{1}-\Phi\left(m, t_{2}\right)_{1} \geq \frac{1}{4}\left(s_{2}-m\right)
$$

while

$$
\Phi\left(m, t_{1}\right)_{1}-\Phi\left(s_{1}, t_{1}\right)_{1} \geq \frac{1}{4}\left(m-s_{1}\right)
$$

and recalling that $\Phi\left(m, t_{1}\right)_{1}=\Phi\left(m, t_{2}\right)_{1}=c(m)_{1}$ and summing, we obtain

$$
\Phi\left(s_{2}, t_{2}\right)_{1}-\Phi\left(s_{1}, t_{1}\right)_{1} \geq \frac{1}{4}\left(s_{2}-s_{1}\right)
$$

We will call tubular coordinates around $c$ the formula (52).

3.5.1. Counterexample. The hypothesis " $c \in C^{2}$ " in the previous proposition may be broadened to " $c \in C^{1,1}$ "; but the

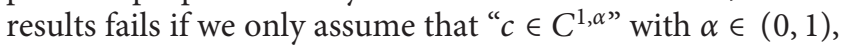
as seen in this example (adapted from [1]).

Example 6. Let $0<\alpha<1$ and $c(\theta)=\left(\theta,|\theta|^{1+\alpha}\right)$; then, for $\theta>0$

$$
c(\theta)+a N(\theta)=\left(\theta-\frac{a(\alpha+1) \theta^{\alpha}}{\sqrt{(\alpha+1)^{2} \theta^{2 \alpha}+1}}, \frac{a}{\sqrt{(\alpha+1)^{2} \theta^{2 \alpha}+1}}+\theta^{\alpha+1}\right),
$$

and this meets the $y$ axes for

$$
a=\tilde{a}(\theta)=\frac{\theta^{1-\alpha} \sqrt{(\alpha+1)^{2} \theta^{2 \alpha}+1}}{\alpha+1} .
$$

So, by symmetry,

$$
c(\theta)+\tilde{a}(\theta) N(\theta)=c(-\theta)+\tilde{a}(-\theta) N(-\theta)
$$

and at the same time,

$$
\lim _{\theta \longrightarrow 0} \widetilde{a}(\theta)=0 .
$$

3.5.2. Nearby Points. Suppose $c: S^{1} \longrightarrow \mathbb{R}^{2}$ is a $C^{2}$ immersed curve. Let $0<\tau \leq \tau_{c}$ and $V_{\tau}=S^{1} \times[-\tau, \tau]$ and

$$
\begin{aligned}
& \Phi: V_{\tau} \longrightarrow \mathbb{R}^{2}, \\
& \Phi(s, t)=c(s)+t N(s),
\end{aligned}
$$

and $U_{\tau}=\Phi\left(V_{\tau}\right)$.

Proposition 8. $U_{\tau}$ is also the set of points at distance at most $\tau$ from the trace $c\left(S^{1}\right)$.

Proof. Let $K=c\left(S^{1}\right)$ be the trace of the curve (it is a compact subset of $\mathbb{R}^{2}$ ). We use the distance function $d_{K}: \mathbb{R}^{2} \longrightarrow \mathbb{R}$ defined as

$$
d_{K}(x):=\inf _{y \in K}|x-y|,
$$

(for an introduction to this object, see [14] and references therein).

Let $x \in U_{\tau}$, there is a $\theta, t \in V_{\tau}$ such that

$$
x=c(\theta)+t N(\theta) \text {. }
$$

So,

$$
|x-c(\theta)| \leq|t| \leq \tau
$$

then let $\tilde{\theta}$ be a minimum for

$$
|x-c(\theta)| \text {. }
$$

So, clearly,

$$
d_{K}(x)=|x-c(\tilde{\theta})| \leq \tau .
$$

Vice versa if $d_{K}(x) \leq \tau$ let $\tilde{\theta}$ be a minimum as above, then geometrical considerations tell that the segment from $x$ to $c(\widetilde{\theta})$ is orthogonal to the tangent $T(\widetilde{\theta})$ at $c(\widetilde{\theta})$.

As a corollary of Proposition 7, for any such neighborhood of the image of $c$, the "projection to $c$ " is a $C^{1}$ multivalued map (with finitely many projections in $S^{1}$ ).

3.6. Not a Covering Map. By looking at the previous Proposition 7, we may think that $\Phi$ is the universal covering map of $U=\Phi(V)$ (see [17] for the definition). This would be very convenient, and indeed, we could use the lifting lemma to ease some of the following proofs. 
Suppose $\tilde{c}, c: S^{1} \longrightarrow \mathbb{R}^{2}$ are $C^{2}$ immersed curves. Consider this statement, that is usually called lifting lemma:

If the trace of $\widetilde{c}$ is contained in $U_{\tau}$, then there is a choice of continuous $\varphi: \mathbb{R} \longrightarrow \mathbb{R}, a: \mathbb{R} \longrightarrow[-\tau, \tau]$ such that

$$
\widetilde{c}(\theta)=\Phi(\varphi(\theta), a(\theta))=c(\varphi(\theta))+a(\theta) N(\varphi(\theta)), \quad \forall \theta .
$$

Unfortunately, this is not the case, as seen in this example in Figure 6, where the curve $c$ is blue and the curve $\widetilde{c}$ is red. The trace of the curve $\tilde{c}$ is all contained in the open set $U_{\tau_{c}}$, but representation (72) cannot hold. We can though prove a version of the lifting lemma useful in the following.

\subsection{Neighborhoods}

\subsubsection{Nearby Projection}

Lemma 5 (nearby projection). Fix a $C^{R}$ immersed curve $c$, with $R \geq 2$.

(1) If $x \in \mathbb{R}^{2}$ and $\widetilde{\sigma} \in S^{1}$ and

$$
d \stackrel{\text { def }}{=}|x-c(\widetilde{\sigma})|<\frac{\delta_{c}}{4}
$$

then there is an $a \in \mathbb{R}$ with $|a| \leq d$ and $a \sigma \in S^{1}$ with

$$
\text { len } c_{\mid[\sigma, \tilde{\sigma}]} \leq 4 d \text {, }
$$

such that

$$
x=c(\sigma)+a N(\sigma)
$$

Note also that $a$ is uniquely identified by $\sigma$.

(2) They are unique in the family of $\sigma$, a such that $|a| \leq \tau_{c}$ and

$$
\operatorname{len} c_{\mid[\sigma, \widetilde{\sigma}]} \leq \delta_{c}
$$

so we can see $\sigma, a$ as functions of $x$, as follows.

(3) Consider $\tilde{x} \in \mathbb{R}^{2}$ and $\widetilde{\sigma} \in S^{1}$ for which

$$
d \stackrel{\text { def }}{=}|\widetilde{x}-c(\widetilde{\sigma})| \leq \frac{\tau_{c}}{2},
$$

let $\varepsilon>0$ small such that $d+\varepsilon<\delta_{c} / 4$ and let $B=B(\tilde{x}, \varepsilon)=$ $\left\{x \in \mathbb{R}^{2}:|x-\tilde{x}|<\varepsilon\right\}$ for convenience. There is a choice of function $a, \varphi: B \longrightarrow \mathbb{R}$ of class $C^{R-1}$ such that

$$
x=\Phi(\varphi(x), a(x))=c(\varphi(x))+a(x) N(\varphi(x)),
$$

for all $x \in B$, and they are unique as specified above.

Note that $\tau_{c} / 2<\delta_{c} / 4<\tau_{c}$.

Proof. Suppose $c$ is by arc parameter (with no loss of generality as explained in Remark 8); so we write (recalling (32)) $|a-b|$ instead of len $c_{\mid[a, b]}$.

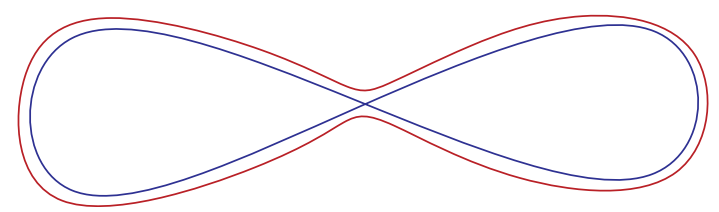

Figure 6: Figure for example in Section 3.6; the curve $c$ is blue and the curve $\tilde{c}$ is red.

(i) Choose $x, \widetilde{\sigma}$ as in the statement and let $J_{\tilde{\sigma}}=\left[\tilde{\sigma}-\delta_{c}, \widetilde{\sigma}+\delta_{c}\right]$, then consider any minimum point $\hat{\theta}$ of

$$
\min _{\theta \in J_{\widetilde{\sigma}}}|x-c(\theta)|
$$

note that the minimum value has to be less than $d$; so,

$$
|c(\widetilde{\sigma})-c(\widehat{\theta})| \leq|c(\widetilde{\sigma})-x|+|x-c(\widehat{\theta})| \leq 2 d,
$$

but at the same time (since $\widetilde{\sigma}$ and $\widehat{\theta}$ are at arc distance at most $\delta_{c}$ ), by the previous Proposition 6 .

$$
|c(\widetilde{\sigma})-c(\widehat{\theta})| \geq \frac{1}{2}|\widehat{\theta}-\widetilde{\sigma}| .
$$

So, combining the two

$$
4 d \geq|\widehat{\theta}-\tilde{\sigma}|
$$

but $4 d<\delta_{c}$ so $\hat{\theta}$ is not at extremes. Then, any $\hat{\theta}$ providing the minimum must be internal in the interval $J_{\tilde{\sigma}}$ : by geometrical reasoning the segment from $x$ to $c(\hat{\theta})$ is orthogonal to the curve so there is a $a$ such that

$$
x=\Phi(\widehat{\theta}, a) .
$$

(ii) Recall that $\delta_{c} / 4 \leq \tau_{c}$; the map $\Phi$ is injective for $\theta \in J_{\tilde{\sigma}}$ and $|a| \leq \tau_{c}$, so $\widehat{\theta}, a$ are unique.

(iii) For any $x \in B$, we have

$$
|x-c(\widetilde{\sigma})|<d+\varepsilon,
$$

and since $d+\varepsilon<\delta_{c} / 4$, then there is an unique $\sigma \in J_{\tilde{\sigma}}$ and $a$ with $|a| \leq \tau_{c}$ such that

$$
x=c(\sigma)+a N(\sigma),
$$

and we denote them by $\sigma=\varphi(x), a=a(x)$. Moreover, we can invert the function

$$
\Phi: J_{\widetilde{\sigma}} \times\left[-\tau_{c}, \tau_{c}\right] \longrightarrow \mathbb{R}^{2}
$$

and write

$$
(\varphi(x), a(x))=\Phi^{-1}(x),
$$

for $x \in B$. This proves that $\varphi, a \in C^{1}(B)$. 


\subsubsection{Global Lifting}

Proposition 9 (global lifting). Suppose $c: S^{1} \longrightarrow \mathbb{R}^{2}$ is a $C^{R}$ immersed curve and $\widetilde{c}: S^{1} \longrightarrow \mathbb{R}^{2}$ is $C^{R-1}$, with $R \geq 2$. Fix $0<\tau<\delta_{c} / 4$. Suppose that we have $|\widetilde{c}(\theta)-c(\theta)| \leq \tau$ for all $\theta$. There exists choice of $a: S^{1} \longrightarrow \mathbb{R}$ and $\varphi: S^{1} \longrightarrow S^{1}$ such that $\tilde{c}(\sigma)=\Phi(\varphi(\sigma), a(\sigma))=c(\varphi(\sigma))+a(\sigma) N_{c}(\varphi(\sigma)), \quad \forall \sigma \in S^{1}$,

with $|a(\sigma)| \leq \tau$ and

$$
\text { len } c_{\mid[\sigma, \varphi(\sigma)]} \leq 4 \tau
$$

holding for all $\sigma$. And, they are unique in the class of $C^{R-1}$ functions such that $|a| \leq \tau_{c}$ and

$$
\operatorname{len} c_{\mid[\sigma, \varphi(\sigma)]} \leq \delta_{c} \text {. }
$$

Note also that $a$ is uniquely identified by $\varphi$.

Proof. We just substitute $x=\widetilde{c}(\sigma)$ in the previous Lemma. By the second point, we can define functions $\varphi(\theta)$ and $a(\theta)$ uniquely as prescribed. By the third point, they are $C^{1}$.

Remark 10. Suppose we are given two curves $c_{1}, c_{2}$ and we know that there exists a choice $(a, \varphi)$ such that

$$
c_{2}(\sigma)=c_{1}(\varphi(\sigma))+a(\sigma) N_{c_{1}}(\varphi(\sigma)), \quad \forall \sigma \in S^{1},
$$

as in (88). If we rotate or translate the two curves, then the above relation will hold, with the same $(a, \varphi)$. If we rescale the two curves by $\lambda>0$, then the relation will hold with $(\lambda a, \varphi)$.

If we choose $\psi \in \operatorname{Diff}^{+}\left(S^{1}\right)$ and we reparameterize all curves at the same time by $\widetilde{c}_{i}=c_{i} \circ \psi$, then

$$
\widetilde{c}_{2}(s)=\widetilde{c}_{1}(\widetilde{\varphi}(s))+\widetilde{a}(s) N_{\widetilde{c}_{1}}(\widetilde{\varphi}(s)), \quad \forall s \in S^{1},
$$

holds for

$$
\begin{aligned}
& \tilde{a}=a \circ \psi, \\
& \widetilde{\varphi}=\psi^{-1} \circ \varphi \circ \psi .
\end{aligned}
$$

This follows from direct computation and Remark 5.

Example 7. So far so good, but $\varphi$ may fail to be a diffeomorphism, as in this simple example in Figure 7. where the curve $c$ is blue and the curve $\tilde{c}$ is red.

But some simple lemmas can help.

Lemma 6. Let $0<\alpha<1$. If we have $w, v \in \mathbb{R}^{n}$ such that

$$
|w-v| \leq \alpha|v|
$$

then the angle $\beta$ between $v$ and $w$ satisfies $|\beta| \leq \arcsin (\alpha)$, and moreover,

$$
\begin{aligned}
& (1-\alpha)|v| \leq v \cdot w \leq(1+\alpha)|v|, \\
& (1-\alpha)|v| \leq|w| \leq(1+\alpha)|v|
\end{aligned}
$$

See Figure 8 on the next page.

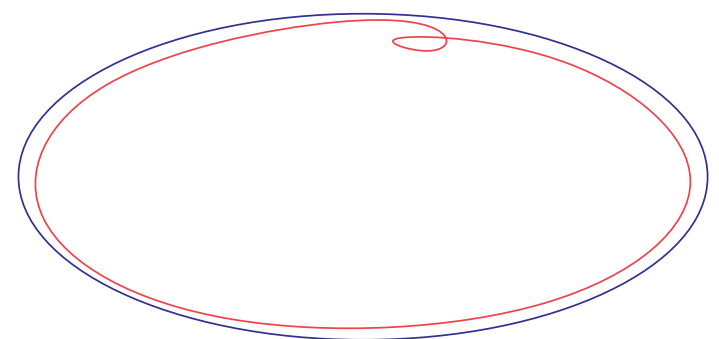

Figure 7: Curves in Example 7; curve $c$ is blue and curve $\widetilde{c}$ is red.

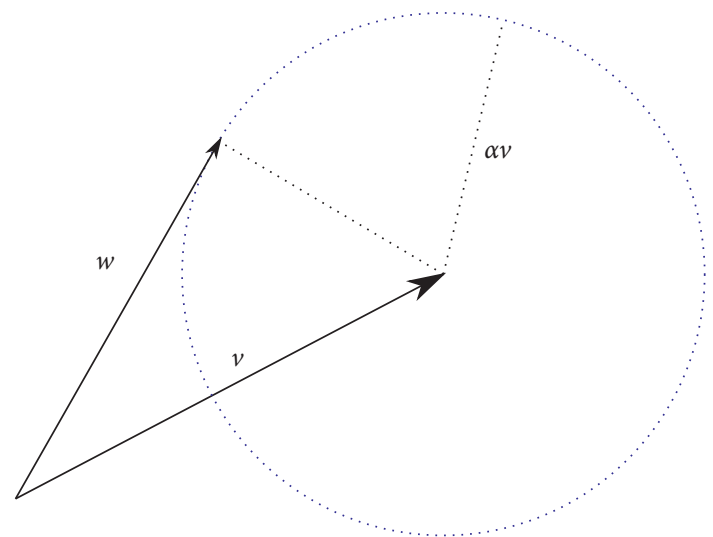

Figure 8: Scheme for Lemma 6.

Remark 11. Let $\alpha>0$. Suppose $c_{1}, c_{2}, c_{3}: S^{1} \longrightarrow \mathbb{R}^{2}$ are $C^{1}$ maps and

$$
\left|c_{2}^{\prime}(\theta)-c_{3}^{\prime}(\theta)\right| \leq \alpha\left|c_{1}^{\prime}(\theta)\right|
$$

if $\psi \in \operatorname{Diff}^{+}\left(S^{1}\right)$ and we reparameterize all curves at the same time by $\widetilde{c}_{i}=c_{i} \circ \psi$, then

$$
\left|\widetilde{c}_{2}^{\prime}(\theta)-\widetilde{c}_{3}^{\prime}(\theta)\right| \leq \alpha\left|\tilde{c}_{3}^{\prime}(\theta)\right|
$$

Similarly, if we rescale, rotate, or translate all curves at the same time.

So, (96) is a geometric estimate, indeed we may rewrite it as

$$
\left|\partial_{c_{1}} c_{2}(\theta)-\partial_{c_{1}} c_{3}(\theta)\right| \leq \alpha,
$$

where $\partial_{c_{1}}=\left(1 /\left|c_{1}^{\prime}\right|\right)(\partial / \partial \theta)$.

Lemma 7. Assume all hypotheses in Proposition 9. Assume moreover that $\tau \leq \tau_{c} / 4$ and assume that

$$
\left|\widetilde{c}^{\prime}(\sigma)-c^{\prime}(\sigma)\right| \leq \frac{1}{2}\left|c^{\prime}(\sigma)\right|, \quad \forall \sigma \in S^{1},
$$

then $\tilde{c}$ is immersed and $\varphi$ is a diffeomorphism. Moreover, when $c$ is parameterized at constant speed, we can state that

$$
\frac{1}{5} \leq \varphi^{\prime} \leq 4
$$

Proof. We rescale and reparameterize $c$ to arc parameter using a reparameterization $\psi$, and at the same time, we 
rescale and reparameterize $\tilde{c}$ using the same rescaling and $\psi$ (note that $\tilde{c}$ is not necessarily by arc parameter); with no loss in generality, as explained in Remarks 10 and 11.

Let $\beta$ be the angle between $\widetilde{c}^{\prime}(\sigma)$ and $c^{\prime}(\sigma)$ : by Lemma 6 , $\beta=\arcsin (\alpha)$, so it is at most $\pi / 6$. Let $\theta=\varphi(\sigma)$; we know that $|\sigma-\theta| \leq 4 \tau \leq \tau_{c}$; the angle $\gamma$ between $c^{\prime}(\sigma)$ and $c^{\prime}(\theta)$ is at most $|\sigma-\theta| \max |k|$ so $\gamma \leq 1 / 2$ : so the angle $\beta+\gamma$ between $\widetilde{c}^{\prime}(\sigma)$ and $\tilde{c}^{\prime}(\varphi(\sigma))$ is at most $1 / 2+\pi / 6$, and this is less than $\pi / 2$.

Deriving in $\sigma$ and assuming that $c$ is by arc parameter

$$
\tilde{c}^{\prime}(\sigma)=T \varphi^{\prime}(\sigma)(1-\kappa a)+a^{\prime} N,
$$

where $T, N, \kappa$ are evaluated at $(\varphi(\sigma))$; then,

$$
\widetilde{c}^{\prime} \cdot T=\varphi^{\prime}(\sigma)(1-\kappa a)
$$

now if $|a| \leq \tau_{c}$ then $(1-\kappa a) \geq 1 / 2$; moreover, by the above reasoning $\widetilde{c}^{\prime} \cdot T>0$, so $\varphi^{\prime}(\sigma)>0$. Moreover, we note that $1 / 2 \leq\left|\vec{c}^{\prime}\right| \leq 3 / 2, \quad 1 / 2 \leq(1-\kappa a) \leq 3 / 2$ and $\cos (1 / 2+\pi / 6) \leq$ $6 / 10$ to prove (100). Relation (93) tells then that $\varphi$ will always be a diffeomorphism, for any curve satisfying the hypotheses.

We summarize all the above: we show sufficient hypothesis such that $\tilde{c}$ may be represented in tubular coordinates around $c$.

Theorem 3 (representation theorem). Suppose c: $S^{1} \longrightarrow \mathbb{R}^{2}$ is a $C^{R}$ immersed curve and $\widetilde{c}: S^{1} \longrightarrow \mathbb{R}^{2}$ is $C^{R-1}$, with $R \geq 2$. Define $\delta_{c}, \tau_{c}$ as in Definition 17. Fix $0<\tau \leq \tau_{c} / 4$. Suppose that we have $|\widetilde{c}(\theta)-c(\theta)| \leq \tau$ and

$$
\left|\widetilde{c}^{\prime}(\theta)-c^{\prime}(\theta)\right| \leq \frac{\left|c^{\prime}(\theta)\right|}{2},
$$

for all $\theta$.

Then, $\widetilde{c}$ is immersed, there are $\varphi \in \operatorname{Diff}^{+}\left(S^{1}\right)$ and $a: S^{1} \longrightarrow[-\tau, \tau]$ of class $C^{R-1}$ such that

$$
\tilde{c}(\varphi(\theta))=\Phi(\theta, a(\theta))=c(\theta)+a(\theta) N(\theta), \quad \forall \theta,
$$

with $|a(\sigma)| \leq \tau$ and

$$
\operatorname{len} c_{\mid[\sigma, \varphi(\sigma)]} \leq 4 \tau
$$

holding for all $\sigma$.

They are unique in the class of $C^{1}$ functions such that $|a| \leq \tau_{c}$ and

$$
\text { len } c_{\mid[\theta, \varphi(\theta)]} \leq \delta_{c} \text {. }
$$

Note also that $a$ is uniquely identified by $\varphi$.

Proof. We can rescale and reparameterize $c$ to arc parameter, and we rescale and reparameterize $\widetilde{c}$ at the same time $(\widetilde{c}$ will not be by arc parameter in general); as discussed in Remarks 8, 10, and 11, the hypotheses and theses are unaffected by this action. Then we apply all previous results. Just note that

$$
\max _{\theta \in S^{1}}|\theta-\varphi(\theta)|=\max _{\theta \in S^{1}}\left|\theta-\varphi^{-1}(\theta)\right|
$$

for any diffeomorphism.

Remark 12. Actually, rerunning on the above proofs with some patience, we can improve the above thesis a bit. We add to the previous theorem these hypotheses: fix $0<\alpha \leq 1 / 2$ and then $0<\tau \leq \alpha \tau_{c} / 2$ and suppose that we have $|\widetilde{c}(\theta)-c(\theta)| \leq \tau$ and

$$
\left|\tilde{c}^{\prime}(\theta)-c^{\prime}(\theta)\right| \leq \frac{\alpha\left|c^{\prime}(\theta)\right|}{2},
$$

for all $\theta$.

Then, all above thesis hold, moreover, there are two continuous functions $f, g:[0, \infty] \longrightarrow \mathbb{R}$ (independent on $\alpha, \tau)$ with $f(0)=g(0)=1$, such that

$$
f(\alpha) \leq \varphi^{\prime} \leq g(\alpha) \text {. }
$$

3.7.3. Asymmetry. Warning: the previous theorem seems symmetric, but it is not. The caveat is in the constants $\tau_{c}, \delta_{c}$ : it may be the case that they are quite different from $\tau_{c}$, $\delta_{c}$. In Figure 9, we see a piece of the two curves: curve $c$ is blue and is flat; curve $\widetilde{c}$ is red, and it has two inflections points $A, B$ where the tangents are at an angle $\beta$ which is as small as we would like, but then the inflection points can also be so close that the normals will cross before reaching curve $c$. So, while there is an easy way of representing $\widetilde{c}$ using tubular coordinates around $c$, there is no way to find $\tilde{\varphi} \in \operatorname{Diff}\left(S^{1}\right), \tilde{a}$ so as to write

$$
c(\varphi(\theta))=\widetilde{c}(\theta)+\widetilde{a}(\theta) \tilde{N}(\theta)
$$

3.7.4. Vice Versa. We have also a sort of vice versa of the previous Theorem 3.

Proposition 10 (derepresentation). Suppose $c: S^{1} \longrightarrow \mathbb{R}^{2}$ is a $C^{2}$ immersed curve and $c_{1}, c_{2}: S^{1} \longrightarrow \mathbb{R}^{2}$ are given by tubular coordinates

$$
c_{i}(\theta)=\Phi\left(\theta, a_{i}(\theta)\right)=c(\theta)+a_{i}(\theta) N(\theta)
$$

where $i=1,2$ and $a_{1}, a_{2}: S^{1} \longrightarrow \mathbb{R}$ are of class $C^{1}$; define

$$
\begin{aligned}
& \alpha \stackrel{\text { def }}{=}\left\|a_{1}-a_{2}\right\|_{\infty} \max |\kappa|, \\
& \beta \stackrel{\text { def }}{=}\left\|\frac{\partial}{\partial c} a_{1}-\frac{\partial}{\partial c} a_{2}\right\|_{\infty},
\end{aligned}
$$

(where $\kappa$ is the curvature of $c$ ). Then,

$$
\left|c_{1}^{\prime}(\theta)-c_{2}^{\prime}(\theta)\right| \leq\left|c^{\prime}(\theta)\right| \sqrt{\left(\alpha^{2}+\beta^{2}\right)},
$$

and (obviously) $\left\|c_{1}-c_{2}\right\|_{\infty}=2 \alpha \tau_{c}$, for all $\theta$.

Proof. We rescale and reparameterize $c$ by arc parameter, and we rescale and reparameterize $c_{1}, c_{2}$ along with $c$, as explained in Remark 11; this operation is justified by Remarks 8 and 10; in particular, note that $\alpha, \beta$ are scale invariant; then, 


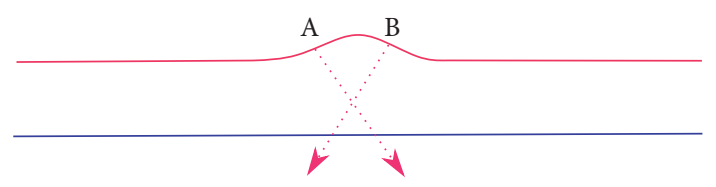

Figure 9: Curves for the example in Section 3.7.3; curve $c$ is blue and curve $\widetilde{c}$ is red.

$$
c_{i}^{\prime}=T\left(1-\kappa a_{i}\right)+a_{i}^{\prime} N
$$

so

$$
c_{1}^{\prime}-c_{2}^{\prime}=-T \kappa\left(a_{1}-a_{2}\right)+\left(a_{1}^{\prime}-a_{2}^{\prime}\right) N .
$$

Hence,

$$
\left|c_{1}^{\prime}-c_{2}^{\prime}\right| \leq \sqrt{\alpha^{2}+\beta^{2}}
$$

3.7.5. Loss of Regularity. Unfortunately, the representation discussed above suffers from a loss of regularity. Indeed, if c: $S^{1} \longrightarrow \mathbb{R}^{2}$ is $C^{2}$ and $a: S^{1} \longrightarrow \mathbb{R}$ is $C^{\infty}$, it may be the case that

$$
\widetilde{c}(\theta)=\Phi(\theta, a(\theta))=c(\theta)+a(\theta) N(\theta),
$$

is of class $C^{1}$ but not of class $C^{2}$.

This can be seen in very simple examples.

Example 8. Suppose that, for $t$ near $t=0$, we have

$$
c(t)= \begin{cases}(t, 0), & t<0, \\ \left(t, \frac{t^{3}}{3}\right), & t \geq 0\end{cases}
$$

such a curve is $C^{2}$ but not $C^{3}$; then, for $t \geq 0$,

$$
\begin{aligned}
T(t) & =\frac{1}{\sqrt{1+t^{4}}}\left(1, t^{2}\right), \\
N(t) & =\frac{1}{\sqrt{1+t^{4}}}\left(-t^{2}, 1\right), \\
\kappa & =\frac{2 t}{\left(1+t^{4}\right)^{3 / 2}},
\end{aligned}
$$

(that can be easily computed using a standard formula for curvature of planar curves, see Sec. 1.7.1 in [21]).

Choose then $a \equiv 1$, so

$$
\widetilde{c}(t)=c(t)+N(t)
$$

but then

$$
\widetilde{c}(t)^{\prime \prime}=c(t)^{\prime \prime}+N(t)^{\prime \prime}
$$

and for $t>0$,

$$
N^{\prime \prime}(t)=\left(\frac{2\left(5 t^{4}-1\right)}{\left(t^{4}+1\right)^{5 / 2}}, \frac{6 t^{2}\left(t^{4}-1\right)}{\left(t^{4}+1\right)^{5 / 2}}\right) .
$$

So,

$$
\lim _{t \longrightarrow 0+} \tilde{c}(t)^{\prime \prime}=(-2,0),
$$

but $\widetilde{c}(t)^{\prime \prime}=0$ for $t<0$.

3.7.6. Neighborhoods of a Curve. The above results encode two different but equivalent ways to define a topology on the "manifold of immersed curves." We specify them by describing the local bases of neighborhoods of a curve $c$.

(i) The "Banach way" in which a local base of open neighborhoods of a curve $c$ is given by the sets

$$
U=U_{c, \varepsilon_{1}}=\left\{\tilde{c}: S^{1} \longrightarrow \mathbb{R}^{2}:\|c-\widetilde{c}\|_{C^{R}}<\varepsilon_{1}\right\},
$$

where $\varepsilon_{1}>0$ is small, and

$$
\|c\|_{C^{R}} \stackrel{\text { def }}{=} \max _{\theta \in S^{1}}|c(\theta)|+\left|c^{\prime}(\theta)\right|+\cdots+\left|c^{(R)}\right| .
$$

(ii) The "geometric way" in which a neighborhood in the local base is defined, for $\varepsilon_{2}>0$ small, as the set $V=$ $V_{c, \varepsilon_{2}}$ of all $\tilde{c}$ that can be expressed as in (88), namely, $\tilde{c}(\sigma)=\Phi(\varphi(\sigma), a(\sigma))=c(\varphi(\sigma))+a(\sigma) N(\varphi(\sigma))$,

for all choices of $a: S^{1} \longrightarrow \mathbb{R}$ and $\phi \in \operatorname{Diff}\left(S^{1}\right)$ with

$$
\begin{array}{r}
\|a\|_{C^{R}}<\varepsilon_{2}, \\
\|\varphi-\mathrm{Id}\|_{C^{R}}<\varepsilon_{2},
\end{array}
$$

where

$$
\begin{aligned}
\|\varphi-\mathrm{Id}\|_{C^{R}}= & \max _{\theta \in S^{1}} d_{S^{1}}(\varphi(\theta), \theta)+\left|\varphi^{\prime}(\theta)-1\right| \\
& +\cdots+\left|\varphi^{(R)}(\theta)\right|,
\end{aligned}
$$

moreover derivatives of $a$ may be computed in arcparameter.

The above are "equivalent" in this sense. Assume that the curve $c$ is $C^{R+1}$.

(i) For any $\varepsilon_{1}$ that defines neighborhood $U$ of the first type, there is small enough $\varepsilon_{2}$ that defines a neighborhood $V$ of the second type, so that $V \subseteq U$; this is easily proved (by using Leibnitz and Faa di Bruno formulas).

(ii) Consider now a neighborhood $V$ of the second type, for an $\varepsilon_{2}>0$ small; for $\varepsilon_{1}$ small enough, the previous results Theorem 3 and Remark 12 tells us that any curve $\widetilde{c} \in U$ can be expressed in tubular coordinates; since tubular coordinates are a local diffeomorphism, similar arguments as above (plus Theorem 2) show that (for $\varepsilon_{1}$ even smaller) $U \subseteq V$.

We skip details for sake of brevity.

In all the above there is, however, an annoying condition: to prove equivalence of $C^{R}$ neighborhoods, we have to assume that the curve $c$ is $C^{R+1}$. For this reason, this works well for defining topologies in the "manifold of smooth immersed curves"; in this case, we will use neighborhoods of 
the first kind (or, respectively, of the second kind) for all $\varepsilon>0$ and all $R$. This is the common approach, see [12].

3.7.7. Local Injectivity. So far, we have considered parametric curves. We have seen in Theorem 3 that we can represent nearby curves in a unique way using tubular coordinates, i.e., the map $\Phi$.

What happens when we consider geometric curves, that is, curves up to parameterization?

Lemma 8 (local injectivity). Let $c$ be a $C^{2}$ freely immersed planar curve. There exists a $r=r_{c}>0$ such that, if

$$
\begin{aligned}
\tilde{c}(s) & \stackrel{\text { def }}{=} c(s)+a(s) N(s), \\
\tilde{c}(\varphi(s)) & =c(s)+b(s) N(s),
\end{aligned}
$$

with

$$
\begin{array}{r}
\|a\|_{\infty} \leq r, \\
\|b\|_{\infty} \leq r, \\
\left\|\frac{\partial a}{\partial c}\right\|_{\infty} \leq \frac{1}{2}, \\
\left\|\frac{\partial b}{\partial c}\right\|_{\infty} \leq \frac{1}{2},
\end{array}
$$

where $(\partial / \partial c)$ is the arc derivation: then $a \equiv b$ and $\varphi$ is the identity.

Proof. If rescale the curves $c, \widetilde{c}$ and the functions $a, b$ by a constant $\lambda>0$, and we rescale the constant $r_{c}$ by the same constant $\lambda$, then all hypotheses and theses are unaffected. So, we can assume with no loss of generality that $c$ has length $2 \pi$.

If we reparameterize $\widehat{c}=c \circ \psi$ then ( $c f$ the relation (93) in Remark 10) the functions $a, b$ are reparameterized as well; having $\widehat{a}=a \circ \psi$, then $(\partial \widehat{a} / \partial \widehat{c})=(\partial a / \partial c) \circ \psi$; and similarly for $b$, again hypotheses and theses are unaffected.

So, we can assume that $c$ is parameterized by arc parameter with no loss of generality.

Suppose that

$$
\widetilde{c}(\theta)=c(s)+a(s) N(s),
$$

with $|a| \leq \tau_{c}$ and $\left|a^{\prime}\right| \leq 1 / 2$, then

$$
\widetilde{c}^{\prime}=T(1-\kappa a)+a^{\prime} N .
$$

So,

$$
\frac{1}{2} \leq\left|\widetilde{c}^{\prime}\right| \leq \sqrt{\frac{9}{4}+\frac{1}{4}} \leq 2 .
$$

Suppose moreover,

$$
\widetilde{c}(\varphi(\theta))=c(s)+b(s) N(s),
$$

with $\left|b^{\prime}\right| \leq 1 / 2$, then

$$
\frac{1}{2} \leq\left|\widetilde{c}^{\prime}\right| \varphi^{\prime} \leq 2
$$

and then

$$
\frac{1}{4} \leq \varphi^{\prime} \leq 4
$$

By contradiction, we may write

$$
\begin{aligned}
\widetilde{c}_{n}(\theta) & =c(s)+a_{n}(s) N(s), \\
\widetilde{c}_{n}\left(\varphi_{n}(s)\right) & =c(s)+b_{n}(s) N(s),
\end{aligned}
$$

with

$$
\begin{aligned}
\left\|a_{n}\right\|_{\infty} & \leq \frac{1}{n}, \\
\left\|b_{n}\right\|_{\infty} & \leq \frac{1}{n}, \\
\left\|a_{n}^{\prime}\right\|_{\infty} & \leq \frac{1}{2}, \\
\left\|b_{n}^{\prime}\right\|_{\infty} & \leq \frac{1}{2},
\end{aligned}
$$

where $\varphi_{n}$ is not the identity: then, when $1 / n<\tau_{c} / 4$, the uniqueness condition (90) is contradicted, so there is a $\sigma_{n}$ such that

$$
\operatorname{len} c_{n \mid\left[\sigma_{n}, \varphi_{n}\left(\sigma_{n}\right)\right]} \geq \delta_{c} \text {. }
$$

So, using Theorem 2,

$$
\liminf _{n \longrightarrow \infty} d_{S^{1}}\left(\varphi_{n}^{-1}\left(\theta_{n}\right), \theta_{n}\right)>0
$$

Up to a subsequence, we can assume that $\theta_{n} \longrightarrow \widetilde{\theta}$ and

$$
\lim _{n \longrightarrow \infty} d_{S^{1}}\left(\varphi_{n}^{-1}\left(\theta_{n}\right), \theta_{n}\right)=\tilde{d}>0 .
$$

We know that

$$
\frac{1}{4} \leq \varphi_{n}^{\prime} \leq 4
$$

up to a subsequence $\varphi_{n} \longrightarrow \varphi$ uniformly and $\varphi_{n}^{-1} \longrightarrow \varphi^{-1}$ uniformly, where $\varphi$ is a bi-Lip homeomorphism. Then, $\tilde{c}_{n} \longrightarrow c$ uniformly, and passing to the limit

$$
c=c \circ \varphi
$$

so $\varphi$ is a diffeomorphism. Moreover,

$$
d_{S^{1}}\left(\varphi^{-1}(\widetilde{\theta}), \widetilde{\theta}\right)=\widetilde{d},
$$

so $\varphi$ cannot be the identity; hence, $c$ is not freely immersed.

3.7.8. Auto Representation (This Section May Be Skipped on a First Read, Since It Is Not Needed in the following). The above result is very important, but the proof gives no hint on what is going on. To this end, we drop the requirement that 
the curve be freely immersed and look at an easy question. Is it possible for a curve to represent itself locally?

Lemma 9. Let $c$ be a $C^{2}$ immersed planar curve. There are only finitely many ways in which the curve can represent itself geometrically, that is, finitely many choices $a, \phi$

$$
c(s)+a(s) N(s)=c(\phi(s)),
$$

with $\phi \in \operatorname{Diff}\left(S^{1}\right)$ and $a: S^{1} \longrightarrow \mathbb{R}$ continuous with $\|a\|_{\infty} \leq \tau_{c} / 2$. In particular, there is a $\rho_{c}>0$ such that, if $\|a\|_{\infty} \leq \rho_{c}$ then $a \equiv 0$.

In particular, if $c$ is freely immersed, then $\|a\|_{\infty} \leq \rho_{c}$ implies $a \equiv 0$ and $\phi \equiv$ Id.

Proof. Define $\delta_{c}, \tau_{c}$ as in Definition 17 (see also Proposition $7)$. Let $\Phi(s, t)=c(s)+t N(s)$. Consider the family $P$ of all the pairs $a, \varphi$ with $\|a\|_{\infty} \leq \tau_{c} / 2$ and $a \neq 0$ and

$$
\Phi(s, a(s))=c(s)+a(s) N(s)=c(\varphi(s))
$$

we will prove that there are only finitely many such pairs.

Hence, we will let $P c$ be smaller than the minimum of $\|a\|_{\infty}$ for all such pairs:

$$
P c=\frac{1}{2} \min _{(a, \varphi) \in P}\|a\|_{\infty} .
$$

In the example, in Figure 10, there are 3 pairs in $P$. We have some very strong properties.

(i) If $\left(a_{1}, \varphi_{1}\right),\left(a_{2}, \varphi_{2}\right) \in P$ and there is a $\widetilde{s} \in S^{1}$ s.t. $\varphi_{1}(\widetilde{s})=\varphi_{2}(\widetilde{s})$, then $\varphi_{1} \equiv \varphi_{2}$ and $a_{1} \equiv a_{2}$. Indeed, there is a small interval $J$ containing $\widetilde{s}$, where we can invert the map $\Phi$ and

$$
\left(s, a_{i}(s)\right)=\Phi^{-1}\left(c\left(\varphi_{i}(s)\right)\right),
$$

that is, the first component of $\Phi^{-1} \circ \mathcal{C}$ is $\varphi_{i}^{-1}$, so $\varphi_{1} \equiv \varphi_{2}$ in $J$; so, the set $\left\{s \in S^{1}: \varphi_{1}(s)=\varphi_{2}(s)\right\}$ is both open and closed. The previous argument also proves that $a_{1} \equiv a_{2}$.

(ii) Figure 11 on the following page can be used as a visual guide in the following proof.

Let $I_{0} \subset S^{1}$ be an open interval such that the length of $\mid c_{I_{0}}$ is less than $\delta_{c}$ and more than $\delta_{c} / 2$. As noted in Remark $9, \delta_{c} \leq \operatorname{len}(c) / 2$. Let $U_{0}$ be the image of $\Phi$ for $s \in I_{0}$ and $|t|<\tau_{c}$. Choose $\left(a_{i}, \varphi_{i}\right) \in P$ with $i \in 1,2$, let

$$
I_{i}=\varphi_{i}\left(I_{0}\right)
$$

we will prove that either $I_{1} \cap I_{2}=\varnothing$, or $I_{1}=I_{2}$, $\varphi_{1} \equiv \varphi_{2}$, and $a_{1} \equiv a_{2}$. Assume that $\widetilde{s} \in I_{1} \cap I_{2}$, let $s_{i}=\varphi_{i}^{-1}(\widetilde{s})$, then $s_{1}, s_{2} \in I_{0}$; so,

$$
c\left(\varphi_{i}\left(s_{i}\right)\right)=c(\widetilde{s}) \text {, }
$$

using the relation (146),

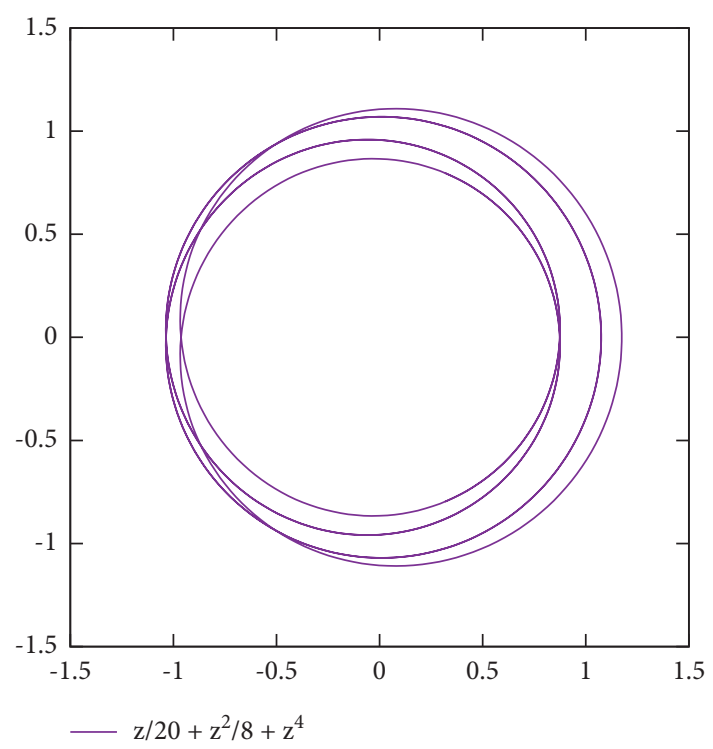

Figure 10: Example of curve with 3 pairs in $P$.

$\Phi\left(s_{i}, a_{i}\left(s_{i}\right)\right)=c\left(s_{i}\right)+a_{i}\left(s_{i}\right) N(s)=c\left(\varphi_{i}\left(s_{i}\right)\right)=c(\widetilde{s}) \in U$,

and the fact that $\Phi$ is a diffeomorphism for $s \in I_{0},|t|<\tau_{c}$ and $c(\widetilde{s}) \in U$, we obtain that $s_{1}=s_{2}=$ $\widehat{s}$ so $\varphi_{1}(\widehat{s})=\varphi_{2}(\widehat{s})=\widetilde{s}$; hence, by the previous point $\varphi_{1} \equiv \varphi_{2}, a_{1} \equiv a_{2}$.

(iii) The differential of $\Phi$ (computed using arc-length derivative) is

$$
T(1-\kappa t) \mathrm{d} s+N \mathrm{~d} t
$$

so the smallest principal value is at least $1 / 2$. We can estimate the length of $c$ for $s \in I_{i}$ to be at least $\delta_{c} / 4$. Hence, there can be only finitely many such intervals.

Example 9. The constants $\rho_{c}$ in Lemma 9 and $r_{c}$ in Lemma 8 though cannot be estimated apriori by using differential quantities such as $\max |\kappa|$. These constants may arbitrarily smaller than the quantity $\tau_{c}$ (defined in Definition 17) that provides the width of the tubular neighborhood (Proposition 7). They really depend on how the curve is drawn.

This is seen in simple examples as follows:

(i) $c(z)=\varepsilon z+z^{2}$ for $z \in S^{1} \subset \mathbb{C}$, or equivalently $c(\theta)=$ $(\varepsilon \cos (\theta)+\cos (2 \theta), \varepsilon \sin (\theta)+\sin (2 \theta)$ for $\theta \in \mathbb{R} /$ $(2 \pi) \sim S^{1}$

that are small $C^{\infty}$ perturbations of the doubly traversed circle $c_{2}$ seen in Example 1; see Figure 12. This curve is freely immersed, but it is quite near to the doubly traversed circle that is not freely immersed. For $\varepsilon$ small, the curvature $\kappa_{c}$ of $c$ is approximatively 1 ; so, $\tau_{c} \sim 1 / 2$, and

$$
\left|c_{2}(\theta)-c(\theta)\right|=\varepsilon,
$$




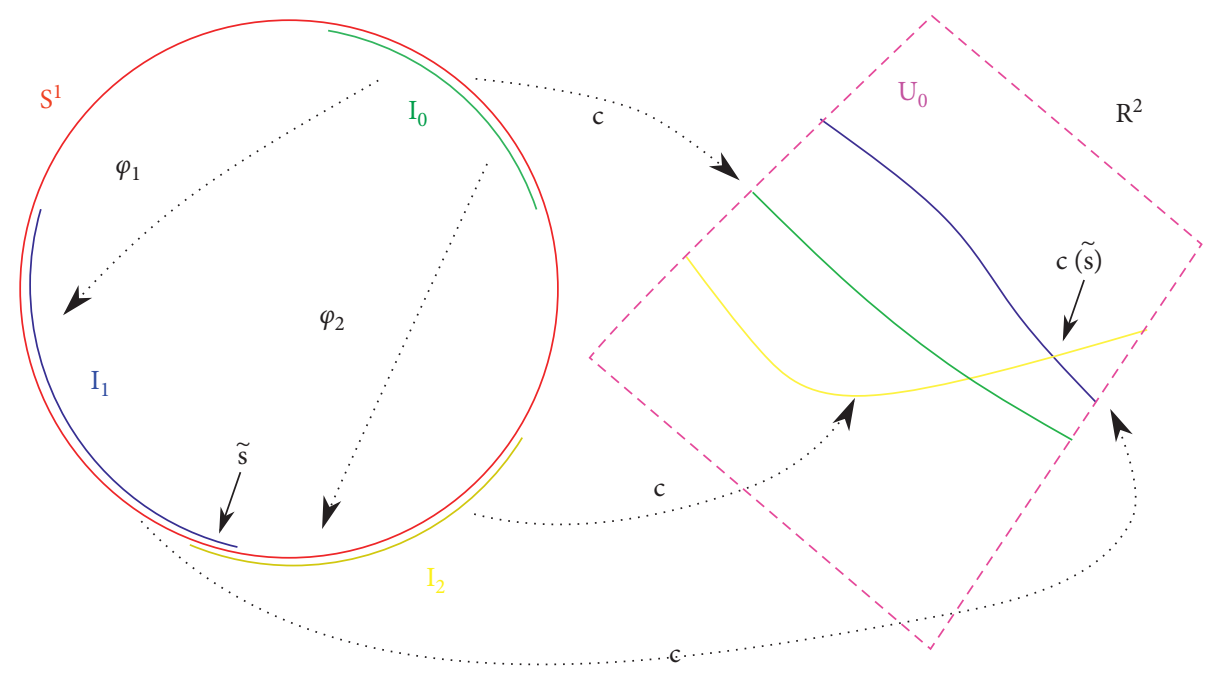

Figure 11: Helper scheme for proof of Lemma 9.

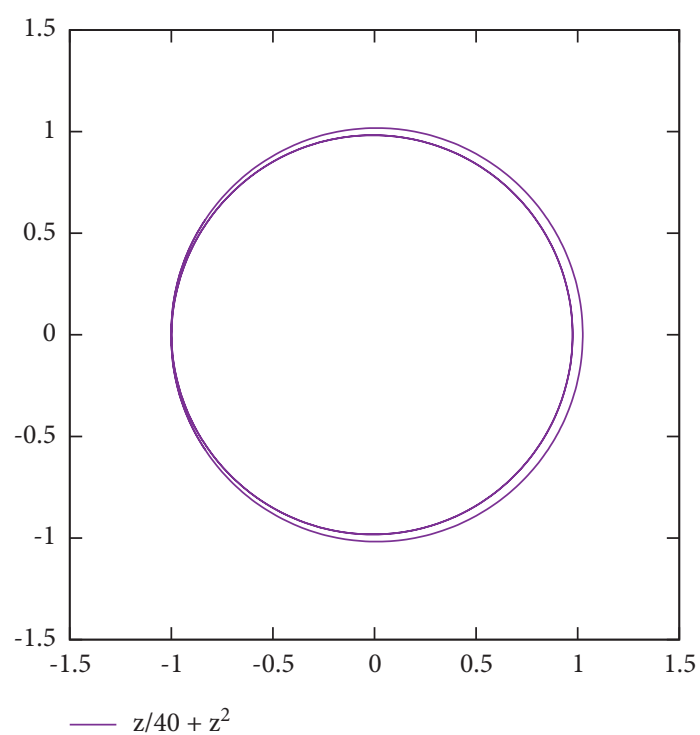

Figure 12: Curve in the Example 9.

while

$$
\begin{aligned}
& \left|c^{\prime}\right| \sim 2 \pm \varepsilon \\
& \left|c_{2}^{\prime}(\theta)-c^{\prime}(\theta)\right|=\varepsilon
\end{aligned}
$$

Hence,

$$
\left|c_{2}^{\prime}(\theta)-c^{\prime}(\theta)\right| \sim \frac{\varepsilon}{2}\left|c^{\prime}(\theta)\right|,
$$

so we can use Theorem 3 to express

$$
c_{2}(\theta)=c(\theta)+a(\theta) N(\theta)
$$

using tubular coordinates when $\varepsilon<1 / 8$, with $a \sim \varepsilon$ : so $r_{c} \widetilde{\leq \varepsilon}$; similarly, we can represent $c(\theta+\pi)$ using $c$, so we have $\rho_{c} \widetilde{\leq \varepsilon}$.
3.8. Free Immersions Are Open. We have thus come to a fundamental result.

Theorem 4. Free immersions are an open subset of immersions.

We can detail and prove this fact in two ways.

(i) We can see it as a corollary of Lemma 8. Consider a neighborhood $V$ defined using the tubular coordinates; precisely, define $V$ as in the second definition in Section 3.7.6, choosing $\varepsilon_{2}<\min \left\{r_{c}, 1 / 2, \tau_{c} / 4\right\}$ and $R=2$; knowing that $\widetilde{c}(s) \equiv \widetilde{c}(\varphi(s))$, we could choose $a \equiv b$, but then by Lemma $8, \varphi$ would be the identity. So, each and any curve in $V$ is freely immersed.

As discussed in Section 3.7.6, this proves the result in the manifold of smooth immersed curves, where the above neighborhoods define a topology.

(ii) If we instead want to prove this for the standard Banach $C^{2}$ topology (first definition in Section 3.7.6), we can proceed as follows. Suppose that $c_{n}$ is a sequence of immersed curves that are not free; and suppose that $c_{n} \longrightarrow c$ in $C^{2}$. We may rescale and reparametrize all the curves so that all have length $2 \pi$ and $\left|c_{n}^{\prime}\right| \equiv 1$, and still $c_{n} \longrightarrow c$ in $C^{2}$; we skip the details (see Theorem 7 in [20]); these assumptions simplify the following arguments. Let $\phi_{n}$ be a sequence such that $c_{n}=c_{n} \circ \phi_{n}$ and $\phi_{n}$ is not the identity; as above, we know that $\phi_{n}^{\prime} \equiv 1$ and we may choose $\phi_{n}$ to be a generator of the isotropy group, so that $\phi_{n}(t)=t+(2 \pi) / j_{n}$; we know that $j_{n}$ is bounded by 4 times the curvature, so up to a subsequence $j_{n}$ is constant, let us call it $j$, and then $\phi_{n}=\phi$ with $\phi(t)=t+(2 \pi) / j$, and passing to the limits $c \circ \phi=c$, so $c$ is not freely immersed. 
Note that both proofs need a compactness argument; this seems unavoidable since the size of the neighborhood cannot be estimated by using differentiable quantities, as explained in Example 9.

\section{The Manifold of Free Geometric Curves}

Definition 18 (classes of curves)

(i) $\operatorname{Imm}\left(S^{1}, \mathbb{R}^{n}\right)$ is the class of immersed curves $c$ : curves such that $c^{\prime} \neq 0$ at all points

(ii) $\operatorname{Imm}_{f}\left(S^{1}, \mathbb{R}^{n}\right)$ is the class of freely immersed curve, the immersed $c$ such that, moreover, if $\phi: S^{1} \longrightarrow S^{1}$ is a diffeomorphism and $c(\phi(t))=c(t)$ for all $t$, then $\phi=\mathrm{Id}$

(iii) $\operatorname{Emb}\left(S^{1}, \mathbb{R}^{n}\right)$ are the embedded curves, maps $c$ that are diffeomorphic onto their image $c\left(S^{1}\right)$; and the image is an embedded submanifold of $\mathbb{R}^{n}$ of dimension 1

Each class contains the one following it (this follows from the propositions seen in Section 2.3).

\subsection{Proof of Theorem 1}

Definition 19

$$
B_{i, f}\left(S^{1}, \mathbb{R}^{2}\right)=\frac{\operatorname{Imm}_{f}\left(S^{1}, \mathbb{R}^{2}\right)}{\operatorname{Diff}+\left(S^{1}\right)},
$$

is the quotient of $\operatorname{Imm}_{f}\left(S^{1}, \mathbb{R}^{2}\right)$ (free immersions) by the positive diffeomorphisms Diff $\left(S^{1}\right)$ (reparameterization).

We now provide the complete proof of Theorem 1, namely, that this $B_{i, f}$ is a manifold, for the case of smooth freely immersed planar curves; afterward, we will show in Section 4.2 how and where the proof in [5] misses some key arguments.

The following proof is for immersed curves $c: S^{1} \longrightarrow \mathbb{R}^{2}$, in a forthcoming paper [15], we will explain how it can be generalized to the case of immersions $\mathscr{I}: M \longrightarrow N$.

4.1.1. Quotient Topology. We discuss the topological aspect of Theorem 1.

Let $\pi: \mathrm{Imm}_{f} \longrightarrow B_{i, f}$ be the canonical projection of the positive quotient that defines $B_{i, f}$ in (157).

The definition of the quotient topology is as follows. A set $\mathscr{Z}$ is open in $B_{i, f}$ when the union of its orbits

$$
\pi^{-1}(\mathscr{Z})=\left\{c \in \operatorname{Imm}_{f}:[c] \in \mathscr{Z}\right\}=\bigcup_{[c] \in \mathscr{Z}}[c],
$$

is open in $\operatorname{Imm}_{f}$, that is, it is open in $C^{\infty}$.

We endow $\operatorname{Imm}_{f}$ with the $C^{\infty}$ topology described earlier and $B_{i, f}$ with the induced quotient topology.

Now, we want to describe a specific family of open neighborhoods that will be quite useful. Fix $c_{1}$ smooth freely immersed. Let $\tau \leq \min \left\{r_{c_{1}}, \tau_{c_{1}} / 4,1 / 2\right\}$, where $r_{c}$ was defined in Lemma 8.
Proposition 11. Consider the set

$$
\mathcal{U}_{c_{1}}=\left\{\widetilde{c} \in \operatorname{Imm}_{f}:\left|\widetilde{c}-c_{1}\right|<\tau,\left|\widetilde{c}^{\prime}-c_{1}^{\prime}\right|<\frac{\left|c_{1}^{\prime}\right|}{3}\right\},
$$

and this set is open in $C^{\infty}\left(S^{1} \longrightarrow \mathbb{R}^{2}\right)$.

Proof. This is a simple case of the arguments of Section 3.7.6. Let $m=\min \left|c_{1}^{\prime}\right|$, let $c_{2} \in \mathscr{U}_{c_{1}}$ and

$$
\begin{aligned}
& \alpha=\left\|c_{2}-c_{1}\right\|_{\infty}, \\
& \beta=\left\|\frac{\partial}{\partial c_{1}} c_{2}-\frac{\partial}{\partial c_{1}} c_{1}\right\|_{\infty},
\end{aligned}
$$

we know that $\alpha<\tau, \beta<1 / 3$; if $c_{3}$ is a smooth curve and satisfies

$$
\begin{aligned}
& \left\|c_{3}-c_{2}\right\|_{\infty}<(\tau-\alpha), \\
& \left\|c_{3}^{\prime}-c_{2}^{\prime}\right\|_{\infty}<\left(\frac{1}{3}-\beta\right) m,
\end{aligned}
$$

then by the results in Section $3 c_{3} \in \mathscr{U}_{c_{1}}$.

By Theorem 4 , all curves in $\mathcal{U}_{c_{1}}$ are freely immersed. define

Now, let us reparameterize all the curves in $\mathcal{U}_{c_{1}}$ and

$$
\mathscr{W}_{c_{1}} \stackrel{\text { def }}{=}\left\{\widetilde{c} \circ \varphi:\left|\widetilde{c}-c_{1}\right|<\tau,\left|\widetilde{c}^{\prime}-c_{1}^{\prime}\right|<\frac{\left|c_{1}^{\prime}\right|}{3}, \varphi \in \operatorname{Diff}^{+}\right\},
$$

since the above conditions are reparameterization invariant, then

$$
\mathscr{W}_{c_{1}}=\bigcup_{c_{2}=c_{1} \circ \varphi, \varphi \in \operatorname{Diff}^{+}} \mathcal{U}_{c_{2}},
$$

that is an union of open sets; hence, it is open in $C^{\infty}$. Moreover, it contains all the orbits of all of its curves: in the language of [5], we may say that " $\mathscr{W}_{c_{1}}$ is saturated for the action of Diff $+\left(S^{1}\right)$."

So, we define

$$
\widetilde{\mathscr{W}}_{c_{1}}=\pi\left(\mathscr{W}_{c_{1}}\right)
$$

and we have

$$
\mathscr{W}_{c_{1}}=\pi^{-1}\left(\widetilde{\mathscr{W}}_{c_{1}}\right)
$$

Hence, $\widetilde{\mathscr{W}}_{c_{1}}$ is open in $B_{i, f}$.

4.1.2. Geometric Representation. We discuss the representation aspect of Theorem 1 . Consider again the set $\mathscr{W}_{c_{1}}$ defined in (162). For any curve $\widetilde{c}$ in this set, by Theorem 3, we have a representation

$$
\widetilde{c} \circ \varphi=c+a N
$$

with $|a| \leq \tau$. 
Let $\widehat{c}=\widetilde{c} \circ \varphi$ for convenience; by the derepresentation result Theorem 10 (setting $\alpha=1 / 8, \beta=1 / 3$ in that proposition and noting $\sqrt{(1 / 64+1 / 9}<1 / 2)$, we have

$$
\left|\widehat{c}^{\prime}(\theta)-c_{1}^{\prime}(\theta)\right|<\left|c_{1}^{\prime}(\theta)\right| \frac{1}{2},
$$

so there is an unique reparameterization of $\widetilde{c}$ that can be expressed in tubular coordinates around $c$; by Lemma 8 , this means that $\varphi$ is uniquely identified by $\widetilde{c}$; so, we will concentrate on $a$.

Proposition 12. Let

$$
\mathbb{Q}_{c_{1}} \stackrel{\text { def }}{=}\left\{a: S^{1} \longrightarrow \mathbb{R}: \exists \widetilde{\mathcal{c}} \in \mathscr{U}_{c_{1}}, \exists \varphi \in \operatorname{Diff}^{+}, \widetilde{c} \circ \varphi=c+a N\right\},
$$

be the set of all such $a$. This set is open.

Proof. The map $(\varphi, a) \mapsto(c+a N) \circ \varphi^{-1}$ is smooth, and $\mathbb{Q}_{c_{1}}$ is the projection on the second component of the counterimage of $\mathscr{W}_{c_{1}}$ that is open.

All of the above can be stated in the language of [5] as follows: the set $\mathscr{W}_{c_{1}}$ is an open neighborhood of $c_{1}$ in $C^{\infty}$, it is composed only of freely immersed curves, it is saturated for the Diff $+\left(S^{1}\right)$-action and the map

$$
(\varphi, a) \mapsto(c+a N) \circ \varphi^{-1},
$$

splits it smoothly as

$$
\mathscr{W}_{c_{1}} \cong Q_{c_{1}} \times \operatorname{Diff}+\left(S^{1}\right) .
$$

4.1.3. Charts. Choose a curve $c_{1}$; consider the map (it is not the same map $\Phi$ defined in Proposition 7)

$$
\begin{aligned}
& \Phi_{c_{1}}: \mathscr{Q}_{c_{1}} \longrightarrow \mathscr{W}_{c_{1}}, \\
& \Phi_{c_{1}}(a) \stackrel{\text { def }}{=}_{c_{1}}+a N_{1},
\end{aligned}
$$

and we already proved in Lemma 8 that it is injective; $\Phi_{c_{1}}$ is also smooth as a map from $C^{\infty}$ to $C^{\infty}$.

If we compose

$$
\widetilde{\Phi}_{c_{1}}=\pi \circ \Phi_{c_{1}}
$$

then the composition

$$
\tilde{\Phi}_{c_{1}}: \mathscr{Q}_{c_{1}} \longrightarrow \widetilde{\mathscr{W}}_{C_{1}},
$$

is bijective: indeed, if $C_{1} \in \widetilde{\mathscr{W}}_{C_{1}}$, then we proved in Section 3 that, picking a $\tilde{c}$ in the equivalence class $C_{1}$,

$$
\tilde{c} \in \mathcal{U}_{c_{1} \circ \varphi},
$$

for an unique $\varphi$, and then

$$
\tilde{c} \circ \varphi^{-1}=c_{1}+a N_{1},
$$

for a unique $a$.
4.1.4. Atlas. To conclude, we discuss the Atlas of charts needed for Theorem 1. For $i=1,2$, consider now two equivalence classes $C_{i} \in B_{i, f}$ and choose a curve $c_{i} \in C_{i}$ in each; we consider the maps

$$
\widetilde{\Phi}_{c_{i}}=\pi \circ \Phi_{c_{i}}
$$

We want to check that these are charts of an Atlas for the manifold.

Suppose that

$$
\widetilde{\mathcal{c}} \in \widetilde{\mathscr{W}}_{c_{1}} \cap \widetilde{\mathscr{W}}_{c_{2}}
$$

then

$$
\begin{aligned}
& \tilde{c}\left(\varphi_{1}(\theta)\right)=c_{1}(\theta)+\widetilde{a}_{1}(\theta) N_{c_{1}}(\theta), \\
& \tilde{c}\left(\varphi_{2}(\theta)\right)=c_{2}(\theta)+\widetilde{a}_{2}(\theta) N_{c_{2}}(\theta),
\end{aligned}
$$

and we need to check that

$$
\widetilde{\Phi}_{c_{2}}^{-1} \circ \widetilde{\Phi}_{c_{1}}
$$

is smooth in a neighborhood of $\tilde{a}_{1}$. We can change variable in the previous one, that is, reparameterize $c_{1}, c_{2}$, so that

$$
\begin{aligned}
& \tilde{c}(\theta)=c_{1}(\theta)+\tilde{a}_{1}(\theta) N_{c_{1}}(\theta), \\
& \tilde{c}(\theta)=c_{2}(\theta)+\tilde{a}_{2}(\theta) N_{c_{2}}(\theta),
\end{aligned}
$$

but for $a_{1}$ near $\tilde{a}_{1}$ we know that

$$
c_{1}(\theta)+a_{1}(\theta) N_{c_{1}}(\theta) \in \mathscr{U}_{c_{2}},
$$

by Lemma 10, so by the representation Theorem 3 , there are $\varphi$ and $a_{2}$ dependent on $a_{1}$ such that

$$
c_{1}(\theta)+a_{1}(\theta) N_{c_{1}}(\theta)=c_{2}(\varphi(\theta))+a_{2}(\varphi(\theta)) N_{c_{2}}(\varphi(\theta)) .
$$

The representation theorem's proof shows that the dependency of $a_{2}$ on $a_{1}$ is smooth (it is given by the inverse of the tubular coordinates, as discussed in the nearby projection Lemma 5).

This concludes the desired proof of Theorem 1 .

4.2. Comparison with [5]. We endow $N$ with a Riemannian metric such that scalar curvatures are bounded and convexity radius is bounded uniformly away from zero; see [7].

The proof in [5] is presented for generic immersions $\mathscr{I}: M \longrightarrow N$; we fix one such immersion.

We follow notations and definitions in [5], here copied for convenience of the user (parts copied from [5] will be in italic, and enclosed in $\ll \ldots \gg)$.

$\ll W e$ choose connected open sets $\left(U_{\alpha}\right)_{\alpha}$ and $\left(W_{\alpha}\right)_{\alpha}$ such that $W_{\alpha} \subset \overline{W_{\alpha}} \subset U_{\alpha} \subset M,\left(W_{\alpha}\right)_{\alpha}$ is an open cover of $M$, each $\overline{W_{\alpha}}$ is compact, $\left(U_{\alpha}\right)_{\alpha}$ is a locally finite open cover of $M$, and such that $\mathscr{I}_{\mid U_{\alpha}}$ is an embedding. $\gg$

$\ll$ Let $p: \mathscr{N}(\mathscr{I}) \longrightarrow M$ be the normal bundle of $\mathscr{I}$, defined in the following way: for $x \in M$, let

$$
\mathcal{N}(\mathscr{I})_{x} \stackrel{\text { def }}{=}\left(T_{x} \mathscr{I}\left(T_{x} M\right)\right)^{\perp},
$$


that is, the orthogonal complement of tangent at $\mathscr{I}(x)$ of the immersed manifold in $T_{\mathscr{I}(x)} N$.»

«The following diagram

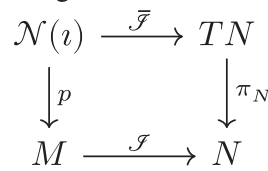

is a vector bundle homomorphism over $\mathscr{I}$, which is fiberwise injective.

$\ll$ Let $\exp ^{N}: T N \longrightarrow N$ be the exponential map on $N$. Now, there is a neighbourhood $U$ of the zero section in the previous bundle $\mathcal{N}(\mathscr{I})$ that is small enough so that

$$
\exp ^{N} \circ \overline{\mathscr{I}}
$$

when restricted to $U U_{\alpha}$ is a diffeomorphism with its image. The restriction of $\exp ^{N} \circ \overline{\mathscr{I}}$ to $U$ is called $\tau^{\mathscr{I}}$. It will serve us as a substitute for a tubular neighborhood of $\mathscr{I}(M)$.»

The notation $U \mid U_{\alpha}$ is not described in [5], but by its usage it should be equivalent to $U \cap p^{-1}\left(U_{\alpha}\right)$. Note also that, later on, the paper adds the superscript $\mathscr{I}$ to $U_{\alpha}$ and will write it as $U_{\alpha}^{\mathscr{J}}$.

Consider planar immersed curves $c: S^{1} \longrightarrow \mathbb{R}^{2}$ : normal vectors at $c(\theta)$ are a one dimensional space $t N_{c}$ (where $N_{c}$ is the normal vector to the curve $c$ as defined in Definition 12); hence, the fibre of $\mathscr{N}(\mathscr{I})$ is one dimensional, so a point in the bundle $\mathcal{N}(\mathscr{I})$ can be represented by a pair $\theta \in S^{1}, t \in \mathbb{R}$, and the map $\tau^{\mathscr{I}}$ becomes the map $\Phi$ defined in (52) in Proposition 7. Fix a $\tau>0$, small, as we will discuss later on. We cover $S^{1}$ by $\operatorname{arcs} U_{\alpha} \subseteq S^{1}$ each shorter than $\varepsilon$, and $W_{\alpha} \subset U_{\alpha}$ subarcs that can be chosen so that they are an open cover; for small $\varepsilon$ we can ensure that tubular coordinates can be perused. The open set $U$ will include normal vectors $t N_{c}$ with $|t|<\varepsilon$.

The statement of main Theorem 1.5 in [5] starts as follows.

Let $\mathscr{I}$ be a free immersion $M \longrightarrow N$. Then, there is an open neighborhood $\mathscr{W}(\mathscr{I})$ in $\operatorname{Imm}(M, N)$ which is saturated for the Diff $(M)$-action and which splits smoothly as

$$
\mathscr{W}(\mathscr{I})=\mathscr{Q}(\mathscr{I}) \times \operatorname{Diff}(M) .
$$

Here, $Q(\mathscr{I})$ is a smooth splitting submanifold of Imm $(M, N)$, diffeomorphic to an open neighborhood of 0 in $C^{\infty}(N(\mathscr{I}))$. In particular, the space $\operatorname{Imm}_{f}(M, N)$ is open in $C^{\infty}(M, N)$.

The proof covers also the case when $M$ is not compact; we will assume that $M$ is compact so that some arguments can be simplified.

The proof goes as follows.

Define

$$
\mathcal{U}(\mathscr{I}) \stackrel{\text { def }}{=}\left\{j \in \operatorname{Imm}(M, N): j\left(W_{\alpha}\right) \subseteq \tau^{\mathscr{I}}\left(U_{\mathscr{I}} \mid U_{\alpha}^{\mathscr{I}}\right) \forall \alpha\right\},
$$

(the proof then goes on showing that this is an open set-we skip details).

For each $j \in U(i)$, we define

$$
\begin{aligned}
& \phi_{i}(j): M \longrightarrow U^{\mathscr{I}} \subseteq N(\mathscr{I}), \\
& \phi_{i}(j)(x) \stackrel{\operatorname{def}}{=}\left(\tau^{\mathcal{I}} \mid\left(U^{\mathcal{I}} \mid U_{\alpha}^{\mathcal{I}}\right)\right)^{-1}(j(x)), \quad \text { if } x \in W_{\alpha}^{\mathscr{I}} .
\end{aligned}
$$

Indeed, we know that $\tau^{\mathscr{I}} \mid\left(U^{\mathscr{I}} \mid U_{\alpha}^{\mathscr{I}}\right)$ is a diffeomorphism onto its image, and that $j(x) \in \tau^{\mathscr{I}}\left(U^{\mathscr{I}} \mid U_{\alpha}^{\mathscr{I}}\right)$ when $x \in W_{\alpha}^{\mathscr{I}}$, by definition of $\mathcal{U}(\mathscr{I})$.

Then $\phi^{\mathscr{I}}: U(\mathscr{I}) \longrightarrow C^{\infty}(M, \mathscr{N}(\mathscr{I}))$ is a mapping which is bijective onto the open set

$$
\mathscr{V}(\mathscr{I}) \stackrel{\text { def }}{=}\left\{h \in C^{\infty}(M, \mathcal{N}(\mathscr{I})): h\left(W_{\alpha}\right) \subseteq U^{\mathscr{I}} \mid U_{\alpha}^{\mathscr{I}}, \forall \alpha\right\},
$$

in $C^{\infty}(M, \mathscr{N}(\mathscr{I}))$. Its inverse is given by the smooth mapping

$$
\tau_{*}^{\mathscr{I}}: h \mapsto \tau^{\mathscr{I}} \circ h
$$

The proof then goes on showing that this $\varphi$ is smooth (we skip details).

We now translate the above objects into the language of Section 4.1. If $c=\mathscr{I}: S^{1} \longrightarrow \mathbb{R}^{2}$ is a freely immersed curve, and $\widetilde{c}=j$ is a curve such that

$$
\widetilde{c}\left(W_{\alpha}\right) \subset U_{\alpha}
$$

then $\|\widetilde{c}-c\| \leq 2 \varepsilon$; so, for $\varepsilon$ small, for each $\sigma \in S^{1}$, there are $\theta \in S^{1}, t \in \mathbb{R}$ such that

$$
\widetilde{c}(\sigma)=c(\theta)+a N_{c}(\theta),
$$

and this pair $\theta \in S^{1}, t \in \mathbb{R}$ is exactly associated to correct point in the above bundle, that is, we can write

$$
\begin{aligned}
x & =\left(\theta, t N_{c}(\theta)\right) \in \mathscr{N}(\mathscr{I}), \\
\tilde{c}(\sigma) & =\tau^{\mathcal{I}}(x) .
\end{aligned}
$$

Possibly reducing the width $\varepsilon$ of the tubular neighborhoods, we can also use Proposition 9 to ensure that the above representation is "unique." So, Proposition 9 can be applied, and this means that, for each $\sigma$, we can write

$$
\widetilde{c}(\sigma)=c(\varphi(\sigma))+a(\sigma) N_{c}(\varphi(\sigma)),
$$

so, in conclusion, we can explicitly write the map $h$ above defined as

$$
h(\theta)=\left(\varphi(\theta), a(\theta) N_{c}(\varphi(\theta))\right),
$$

where the first component in $h$ encodes a position in the base space $M=S^{1}$ of the bundle $\mathscr{N}(\mathscr{I})$, the second encodes a normal vector $a N_{c}$ to the curve $c$ at $\varphi(\theta)$. Indeed, the inverse $\tau^{*}$ is exactly the map (193).

The proof continues as follows.

We have $\tau_{*}^{\mathcal{I}}(h \circ f)=\tau_{*}^{\mathcal{I}}(h) \circ f$ for those $f \in \operatorname{Diff}(M)$ which are near enough to the identity so that $h \circ f \in \mathscr{V}(i)$ (that was defined in (188)). We consider now the open set (we added the notation $\mathscr{Z}$ for ease of reference) 


$$
\mathscr{Z}^{\text {def }}=\{h \circ f: h \in \mathscr{V}(i), f \in \operatorname{Diff}(M)\} \subseteq C^{\infty}\left(\left(M, \mathcal{U}^{i}\right)\right) .
$$

Obviously, we have a smooth mapping from it into

$$
C^{\infty}\left(U^{\mathscr{I}}\right) \times \operatorname{Diff}(M)
$$

given by

$$
h \mapsto\left(h \circ(p \circ h)^{-1}, p \circ h\right),
$$

where $C^{\infty}\left(U^{\mathscr{F}}\right)$ is the space of sections of $U^{i} \longrightarrow M . \gg$

Here comes the first mistake in the original proof. Consider Example 8, where $c=\mathscr{I}$ is the blue curve and $j=\widetilde{c}$ is the red curve. There is a choice of $U_{\alpha}, W_{\alpha}$ such that $j \in \mathcal{U}(\mathscr{I})$. We can express

$$
\widetilde{c}(\sigma)=c(\varphi(\sigma))+a(\sigma) N(\varphi(\sigma))
$$

and then define $h$ by (194); but when we apply the rule (197) to this $h$, we obtain that

$$
p \circ h=\varphi,
$$

and $\varphi$ is a map $\varphi: S^{1} \longrightarrow S^{1}$ that is not a diffeomorphism.

The correct statement is that map (197) achieves a splitting of the open set $\mathscr{Z}$ described in (195) into

$$
C^{\infty}\left(U^{\mathscr{I}}\right) \times C^{\infty}(M \longrightarrow M)
$$

Some conditions must be added to the definition of $\mathscr{Z}$ to make sure that $\varphi$ is a diffeomorphism; as was done in (159) to define $\mathcal{U}$, by adding the condition $\left|\widetilde{c}^{\prime}-c_{1}^{\prime}\right|<\left|c_{1}^{\prime}\right| / 3$ : this condition is necessary to apply the representation Theorem 4. No similar condition is present in the proof in [5].

The proof afterwards proceeds as follows.

So, if we let

$$
\mathbb{Q}(i) \stackrel{\text { def }}{=} \tau_{*}^{\mathscr{I}}\left(C^{\infty}\left(U^{\mathscr{I}}\right) \cap V(\mathscr{I})\right) \subset \operatorname{Imm}(M, N) .
$$

We have

$$
\mathscr{W}(\mathscr{I}) \stackrel{\text { def }}{=} U(\mathscr{I}) \circ \operatorname{Diff}(M) \cong \mathscr{Q}(\mathscr{I}) \times \operatorname{Diff}(M) \cong\left(C^{\infty}\left(U^{\mathscr{I}}\right) \cap \mathscr{V}(\mathscr{I})\right) \times \operatorname{Diff}(M)
$$

since the action of Diff $(M)$ on $(\mathscr{I})$ is free. Consequently, Diff $(M)$ acts freely on each immersion in $\mathscr{W}(\mathscr{I})$, so $\operatorname{Imm}_{f}(M, N)$ is open in $C^{\infty}(M, N)$.

(this $Q(i)$ is not the same as the $Q_{c_{1}}$ defined in (168), but it has the same scope; the latter one is an open neighborhood of 0 in $C^{\infty}(\mathscr{N}(\mathscr{I}))$ )

This is the second mistake in the proof.

Even if we restrict the open set described in (195) by adding a first order condition, so that the map (197) properly splits Q, we have not guarantee that all curves in the associated neighborhood are freely immersed. This is shown in Example 9.

This is why, in our proof, we added the condition $\tau \leq r_{c}$ to the definition of $\mathcal{U}$.

We have shown that the proof in [5] does not prove the desired result.

\section{Data Availability}

No data were used to support this study.

\section{Conflicts of Interest}

The author declares no conflicts of interest.

\section{Acknowledgments}

The author thanks Prof. Kathryn Mann for her help on the properties of $\operatorname{Diff}^{+}\left(S^{1}\right)$.

\section{References}

[1] M. Abate and F. Tovena, Curves and Surfaces. UNITEXT, Springer, Milan, Italy, 2012, https://books.google.it/books? $\mathrm{id}=\mathrm{iwGjNhpkSeQC}$.
[2] A. Banyaga, The Structure of Classical Diffeomorphism Groups. Mathematics and Its Applications, Springer, Berlin, Germany, 2013, https://books.google.it/books?id=ArEJCAAAQBAJ.

[3] A. Blake and M. Isard, Active Contours, Springer-Verlag, 1998.

[4] V. Caselles, R. Kimmel, and G. Sapiro, "Geodesic active contours," in Proceedings of the IEEE Int. Conf. on Computer Visionpp. 694-699, Cambridge, MA, USA, June 1995.

[5] V. Cervera, F. Mascaró, and P. W. Michor, "The action of the diffeomorphism group on the space of immersions," Differential Geometry and Its Applications, vol. 1, no. 4, pp. 391-401, 1991, http://www.sciencedirect.com/science/ article/pii/0926224591900152.

[6] L. D. Cohen, "On active contour models and ballons," Computer Vision, Graphics, and Image Processing: Image Processing, vol. 53, no. 2, 1991.

[7] R. E. Greene, "Complete metrics of bounded curvature on noncompact manifolds," Archiv der Mathematik, vol. 31, no. 1, pp. 89-95, 1978.

[8] M. W. Hirsch, Differential Topology. Graduate Texts in Mathematics, Springer, New York, NY, USA, 1997, https:// books.google.it/books?id=iSvnvOodW18C.

[9] M. Kass, A. Witkin, and D. Terzopoulos, "Snakes: active contour models," International Journal of Computer Vision, vol. 1 , no. 4, pp. 321-331, 1988.

[10] J. L. Kelley and I. Namioka, Linear Topological Spaces, Springer-Verlag, New York, NY, USA, With the collaboration of W. F. Donoghue, Jr., K. R. Lucas, B. J. Pettis, E. Thue Poulsen, G. B. Price, W. Robertson, W. R. Scott, and K. T. Smith, Second corrected printing, Graduate Texts in Mathematics, No. 36, 1976.

[11] S. Kichenassamy, A. Kumar, P. Olver, A. Tannenbaum, and A. Yezzi, "Gradient flows and geometric active contour models," in Proceedings of the IEEE International Conference on Computer Vision, pp. 810-815, Cambridge, MA, USA, June 1995. 
[12] A. Kriegl and P. W. Michor, "The convenient setting of global analysis," Volume 53 of Mathematical Surveys and Monographs, American Mathematical Society, Providence, RI, USA, 1997.

[13] K. Mann, "A short proof thatDiff ${ }_{c}(M)$ is perfect," The New York Journal of Mathematics, vol. 22, pp. 49-55, 2016, https:// math.berkeley.edu/kpmann/perfectness2.pdf.

[14] C. Mantegazza and A. C. Mennucci, "Hamilton-Jacobi equations and distance functions on Riemannian manifolds," Applied Mathematics and Optimization, vol. 47, no. 1, pp. 125, 2002.

[15] A. C. G. Mennucci, "Topologies and manifolds of immersions," 2021, https://cvgmt.sns.it/person/109/.

[16] P. Michor and D. Mumford, "Riemannian geometries on spaces of plane curves," Journal of the European Mathematical Society, vol. 8, pp. 1-48, 2006.

[17] J. R. Munkres, Topology. Featured Titles for TopologyPrenticeHall, Hoboken, NJ, USA, 2000, https://books.google.it/books? id=XjoZAQAAIAAJ.

[18] R. Walter, Functional Analysis, McGraw-Hill, New York, NY, USA, 1973.

[19] W. Thurston, "Foliations and groups of diffeomorphisms," Bulletin of the American Mathematical Society, vol. 80, no. 2, pp. 304-308, 1974.

[20] A. B. Tumpach and S. C. Preston, "Quotient elastic metrics on the manifold of arc-length parameterized plane curves," Journal of Geometric Mechanics, vol. 9, no. 2, pp. 227-256, 2017, http://aimsciences.org/journals/displayArticlesnew.jsp? paperID=14088.

[21] L. Younes, Shapes and Diffeomorphisms. Applied Mathematical Sciences, Springer, Berlin, Germany, 2019, https://books.google. it/books?id=JwiZDwAAQBAJ ISBN 9783662584965. URL. 